\title{
Effect of combined translation and torsion on undrained uplift capacity of plate anchors: plastic limit analysis (PLA) solution \\ Hamid Reza Nouri ${ }^{1}$, Giovanna Biscontin ${ }^{2}$ and Charles P. Aubeny, M. ASCE ${ }^{3}$
}

\begin{abstract}
Uplift capacity of plate anchors have been the focus of numerous studies, since anchor plates are designed for pull-out in normal operating conditions. However, the response of plate anchors under six-degrees-of-freedom loading caused during extreme loading conditions is poorly understood. The purpose of this study is to propose a simple yet sufficiently accurate analytical solution to investigate the behavior of plate anchor under combined in-plane translation and torsion and to evaluate its effect on the plate uplift bearing capacity. To this end, a modified plastic limit analysis (PLA) approach is introduced and compared with limit equilibrium (LE) and simplified upper bound baseline solutions. The proposed method is verified with three dimensional finite element (3D-FE). The variables considered in this study include plate aspect ratio, plate thickness, as well as load direction and eccentricity. Results of analytical solutions indicate the insensitivity of the "shape" of the shear-torsion yield envelope to plate thickness. This finding facilitates the use of simplified yet reasonable yield envelope for infinitely thin plate obtained from simplified PLA approach for other plate thicknesses. The "size” of the failure envelope (controlled by pure torsional and translational capacity) could be predicted fairly accurately by PLA and LE methods. Combination of these analytical methods offers a simple yet reasonably accurate solution to describe shear-torsion response of anchor plate. The obtained shear-torsion yield envelope is then fitted in the generalized six-degrees-of-freedom yield surface which describes the reducing effect of moment, torsion, and planar forces on the uplift capacity of plate.
\end{abstract}

KEY WORDS: plate anchor, torsional and translational bearing capacity, plastic limit analysis, limit equilibrium, finite element analysis, yield envelope.

\footnotetext{
${ }^{1}$ Senior Geotechnical Engineer, Shannon and Wilson, Inc., 400 North 34 ${ }^{\text {th }}$ Street Suite 100, P.O. Box 300303 Seattle, Washington, USA 98103. Ph.: +1 (206) 695-6924, E-mail: Nouri.hreza@gmail.com; hrn@shanwil.com ${ }^{2}$ University Lecturer in Geotechnical Engineering, University of Cambridge, Department of Engineering, Schofield Centre, High Cross, Madingley Road, Cambridge, UK CB3 0EL. Ph.: +44 (1223) 768-044, E-mail: gb479@cam.ac.uk ${ }^{3}$ Professor, Texas A\&M University, Civil Engineering CE/TTI, 3136 TAMU, College Station, TX 77843-3136, USA. Ph.: (979) 845-4478, E-mail: caubeny@civil.tamu.edu
} 


\section{INTRODUCTION}

2 In recent years the growing trend in searching and developing hydrocarbon reserves has forced

3 offshore oil and gas industry into deep and ultra deep water. It becomes increasingly difficult and

4 costly to keep the natural period of traditional fixed jacket or gravity-based structures below the

5 dominant frequency of the sea wave spectrum in water depths exceeding $500 \mathrm{~m}$ (Aubeny et al.

6 2001). Thus, floating structures anchored to the seabed using catenary or taut-wire moorings has

7 taken the place of conventional platforms (Aubeny et al. 2001). Plate anchors are increasingly

8 being used to moor large floating offshore structures in deep and ultra deep water.

9 Plate anchors are installed to impart substantial uplift loading in normal operating conditions.

10 However, they could be subjected to six-degrees-of-freedom general loading as well. The recent

11 hurricane events in the Gulf of Mexico resulted in partial failure and drift of 17 deepwater

12 Mobile Offshore Drilling Units (MODUs) in hurricanes Ivan, Katrina, and Rita. The drift of the

13 platform causes a significant change in the orientation and amount of the resultant force, which

14 appears as a substantial out-of-plane force acting on the anchor of the intact line. This could lead

15 to substantial reduction in uplift capacity of the plate, failure of the anchor, and catastrophic

16 damages to adjacent oil and gas infrastructure through colliding with other exploration and

17 production platforms and rupturing the network of oil and gas pipelines by dragging anchors of

18 the failed line on the seabed. Importance of understanding the plate anchor response under out-

19 of-plane loading is not limited to design considerations under extreme loading conditions. It is a

20 frequent practice for operators to work on two close wells in the same field. Instead of relocating

21 the drilling unit and its mooring system, it is more efficient to winch between wells on one

22 mooring system without removing and reinstalling anchors. If the wells are far enough, anchors

23 will be subjected to out-of-plane loading. 
24 The undrained uplift bearing capacity of deeply embedded plates has been the focus of the

25 majority of previous studies, while the plate bearing capacity under general loading conditions

26 has received considerably less attention. The purpose of this study is to develop adequately

27 accurate yet simple analytical solutions to quantify the decreasing effect of translation and

28 torsion on pull-out bearing capacity of plate anchors. These simplified approaches are more

29 attractive solutions for practical purposes compared to expensive numerical simulation.

30 This study idealizes the geometry of the plate anchor as a rectangular plate of length $L$, width $W$

31 and thickness $t$ subjected to eccentric loading (Fig. 1): $L$ is taken as the shorter dimension,

32 aligned with the $x$-axis. Also assuming a homogeneous condition for soil, any spatial variation of

33 soil parameters is neglected in this study (i.e. constant undrained shear strength). In general, any

34 eccentricity angle $\psi$ or load angle $\gamma$ may occur. First, a number of simple baseline solutions for

35 pure translation $\left(e=0\right.$ or $\left.\gamma=90^{\circ}\right)$, and pure torsion $\left(\gamma \neq 90^{\circ}\right.$ and $\left.e=\infty\right)$ are derived from limit

36 equilibrium (LE). For general conditions of eccentricity and load orientation for infinitely thin

37 plate, a semi-analytical upper-bound limit analysis based approach is proposed. For a plate of

38 finite thickness under general eccentric load, a more powerful method is required. To this end,

39 the upper-bound plastic limit analysis (PLA) proposed by Yang et al. (2010) for infinitely thin

40 plates is adopted and modified to better predict the bearing capacity for plates with finite

41 thickness. The accuracy of the baseline solutions and PLA approach are evaluated through

42 comparisons to three-dimensional finite element solutions. The proposed analytical solutions are

43 used to develop a simplified approach to incorporate the shear-torsion interaction equation in the

44 generalized yield envelope for plate anchor under six-degrees-of-freedom loading condition. The

45 complete generalized yield surface is used to evaluate the uplift capacity of anchor plates under

46 combined eccentric translational/planar forces and out-of-plane moments. 


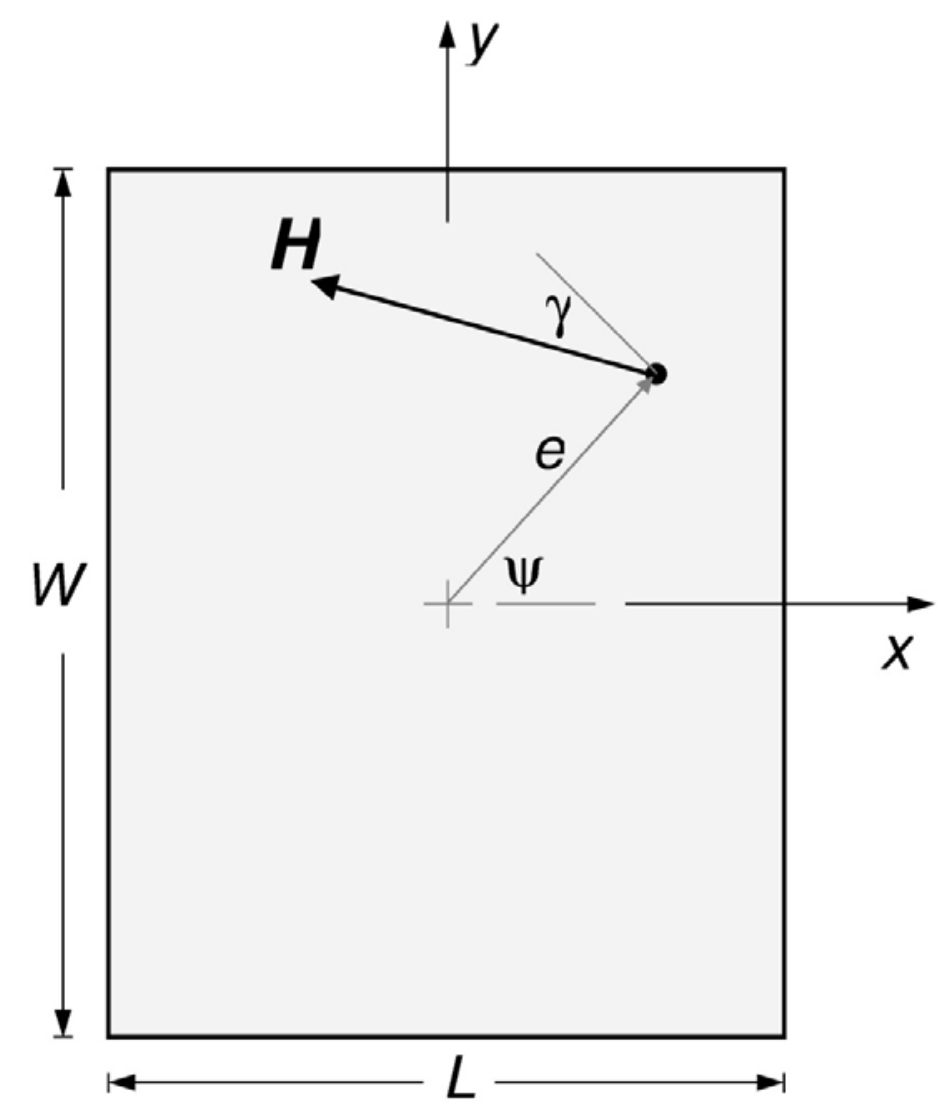

Figure 1- Schematic of eccentrically loaded plate

\section{BACKGROUND}

48 The majority of previous studies concerning the bearing capacity of plates are focused on

49 undrained uplift capacity and limited literature is available on the bearing capacity of plate

50 anchors subjected to general loading condition. O’Neill et al. (2003) used two-dimensional finite

51 element (2D-FE) analysis to investigate the behavior of rectangular and wedge-shaped strip

52 anchors subjected to combined translational, vertical, and rotational loadings. They also

53 developed plastic upper bound solutions to evaluate plane strain ultimate parallel, normal, and

54 rotational plate capacity factors to validate the FE results. They produced FE based yield

55 surfaces and developed an approach to predict the kinematics and trajectory of drag anchor

56 during the installation. Murff et al. (2005) used 2D-FE to develop yield loci for plates of 
57 different thickness and roughness. Yang et al. (2010) employed 3D-FE, as well as plastic upper

58 bound limit analysis, to study the behavior of infinitely thin plates subjected to six-degrees-of-

59 freedom loading. They also introduced a plasticity solution to determine yield loci of an

60 infinitely thin plate under combined translation-torsion. They developed equations to calculate

61 pure sliding and torsional bearing capacity. Nouri (2013) conducted 3D-FE analysis to evaluate

62 the response of plate anchors with finite thickness under in-plane two-way translation and

63 torsion. Their 3D-FE results indicated the insensitivity of the shape of yield envelope (i.e. plate

64 interaction response under combined loading) to thickness variations of square and rectangular

65 anchors.

66 Yang et al. (2010) Upper Bound PLA Solution: Overview

67 Yang et al. (2010) developed an upper bound plastic limit analysis (PLA) formulation for deeply

68 embedded square and rectangular plate anchors under combination of co-planar sliding and

69 torsion. They assumed deep embedment for the plate anchor, which ensures "no separation" of

70 undrained cohesive soil in contact with the foundation. The full attachment of the foundation and

71 soil complies with the normality concept (Tan, 1990) which is essential to apply the limit

72 analysis approach in soils (Chen and Liu, 1990). This assumption seems valid since due to the

73 low permeability of clays in combination with a high loading rate, suction will be generated on

74 faces of the plate (Wang et al. 2010).

75 In their approach a plate is assumed subjected to a force, $F$, in the plane parallel to the plate

76 faces, passing through its center (Fig. 2). If the plate is subjected to a virtual rotation rate, $\dot{\beta}$,

77 around point $\mathrm{O}\left(x_{o}, y_{0}\right), F$ could be calculated by equating the work done by external force, $F$, and

78 the total rate of energy dissipation in the vicinity of plate failure: 


$$
F=\frac{\dot{D}_{s}+\dot{D}_{e}}{\left[\left(x_{f}-x_{o}\right) \cos \phi+\left(y_{f}-y_{o}\right) \sin \phi\right] \dot{\beta}}
$$

79 where the total rate of energy dissipation is equal to the rate of energy dissipated by sliding along

80 the top and bottom surfaces of the plate $\left(\dot{D}_{s}\right)$ in addition to the rate of energy dissipated by the

81 soil resistance on the plate edges $\left(\dot{D}_{e}\right)$ (see Appendix A), and $\phi=\psi+\gamma$ is the angle between line of

82 action of the external force and $y$-axis. The minimum value for the force, $F$, can be determined

83 by optimizing the kinematics of the plate rigid body motions with respect to the coordinates of

84 the rotation center of the rotating plate, $\mathrm{O}\left(x_{0}, y_{0}\right)$. The pure sliding (shear loading) occurs when

85 the line of action of the force, $F$, is passing through the center of the plate and pure torsion

86 occurs when the eccentricity of the line of action of the force, $F$, with respect to center of the

87 plate is infinity $(e \cdot \cos (\gamma)=\infty)$. The rate of energy dissipated along an arbitrary element of $d x$ by

$88 d y$ on top or bottom surfaces of the anchor plate with center at $(x, y)$ and distance of

$89 R(x, y)=\sqrt{\left(x-x_{0}\right)^{2}+\left(y-y_{0}\right)^{2}+(t / 2)^{2}}$ from the center of rotation (Fig. 2(a)), is

$90 d \dot{D}_{s}=\alpha s_{u} R(x, y) \dot{\beta} d x d y$ which can be integrated over the plate area (see Appendix A). The

91 undrained shear strength, $s_{u}$, along top and bottom plate surfaces is assumed to be fully

92 developed in Yang et al. (2010) formulation. The adhesion factor ( $\alpha$ ) for fully bonded plate and

93 soil is assumed to be unity. The second dissipation term $\left(\dot{D}_{e}\right)$ due to the resistance of the soil on

94 the four ends of the plate could be resolved into sliding $\left(F_{\text {st }}\right)$ and normal $\left(F_{\text {nt }}\right)$ components (Fig.

95 2(b)). As these terms are dependent on the plate thickness, they vanish for infinitely thin plates.

96 Yang et al. (2010) assumes that both the normal $\left(F_{\mathrm{nt}}\right)$ and sliding $\left(F_{\mathrm{st}}\right)$ soil resistances on plate

97 ends are fully mobilized at failure. Thus, no interaction is assumed between normal and

98 tangential forces along the plate edges. 


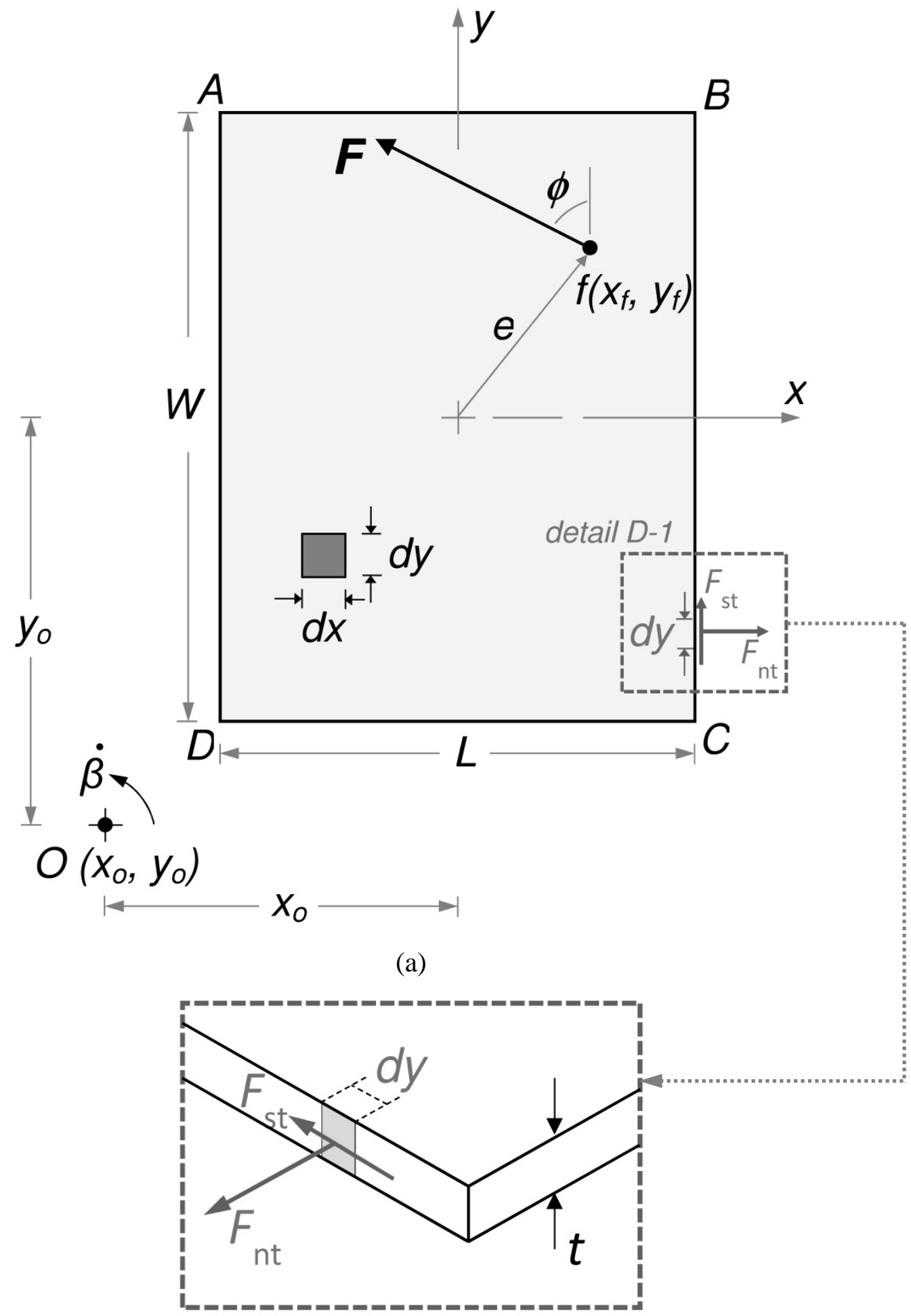

(b)

Figure 2- Upper bound PLA mechanism for shear-torsion loading of a plate anchor: (a) plan view of the plate and mechanism; (b) Detail D-1: isometric view of the plate edge and acting normal $\left(F_{\text {nt }}\right)$ and tangential $\left(F_{\text {st }}\right)$ forces 
99 Assuming an arbitrary element of $t$ (plate thickness) by $d l(=d x$ and $d y$ for edges parallel to $x$ and

$100 y$ axis respectively) on each edges of the plate (Fig. 2), the sliding and normal components are

101 calculated (i.e. $d F_{s t}=\alpha \cdot s_{u} . t . d l$ and $d F_{n t}=N_{e} \cdot s_{u} \cdot t \cdot d l$ ). $N_{e}$ is a simple plane strain bearing

102 capacity factor for each element to determine the normal component and is assumed equal to

$103 N_{e}=7.5$ (O'Neill et al. 2003). The adhesion factor $(\alpha)$ of unity is also adopted for fully attached

104 plate and soil. Total values of normal and tangential portions of soil resistance on the plate edges

105 are determined by integrating along each plate edge (see Appendix A).

106 By substituting the dissipation terms in Eq. 1 and canceling out the angular velocity, $\dot{\beta}$, an

107 expression is obtained in terms of $x_{o}$ and $y_{o}$ for external force, $F$. A least upper bound is obtained

108 by minimizing $F$ with respect to the rotation coordinates $\left(x_{o}, y_{o}\right)$.

109 Issues with the existing Yang et al. (2010) PLA Solution

110 Tables 1 and 2 summarize the results of the Yang et al. (2010) PLA solutions as well as the 3D-

111 FE developed by Nouri (2013) for the ultimate shear and torsion bearing capacity of the square

112 and rectangular $(W / L=2)$ plates of various thicknesses $(t / L=0,1 / 20,1 / 14,1 / 10$, and $1 / 7)$. The

113 Yang et al. (2010) PLA solution developed by Yang et al. (2010) seem to reasonably predict the

114 ultimate shear bearing capacity $\left(N_{s, \max }\right)$ regarding its satisfactory agreement with the 3D-FE

115 results for plate of different geometry. However, the unconservative over-prediction of the 3D-

116 FE results for maximum torsional resistance $\left(N_{t, \max }\right)$ by the PLA solution and the growing

117 difference for the thicker plates suggest the inaccuracy of the current PLA formulation to predict

118 torsional resistance for plates of larger thickness.

119 Fig. 3 plots present the PLA and 3D-FE derived normalized shear-torsion interaction curve for 120 rectangular $(W / L=2)$ plates. Comparison of $H_{x} / H_{x, \max }-T / T_{\max }$ yield envelopes in Fig. 3(a)

121 indicates the unconservative tendency of the PLA approach to over-predict the bearing capacity 
122 by $20-30 \%$. The over-prediction trend is also repeated in PLA derived $H_{y} / H_{y, \max }-T / T_{\max }$

123 interaction curves for rectangular and square plates (Figs. 3(b) and 4, respectively). PLA

124 envelopes show a vertical non-interacting portion for low values of torsional resistance

125 (T/T $\left.T_{\max }<0.3-0.4\right)$, which is not observed in 3D-FE envelopes. This vertical section indicates that

126 the PLA approach predicts no change of the shear resistance by the plate torsion which ends in

127 overestimating the bearing capacity values. This non-interacting vertical portion in the PLA

128 envelopes shortens for the plates of smaller thickness and eventually vanishes for infinitely thin

129 plate $(t=0)$, since the resistance for thinner plates is solely controlled by the energy dissipated on

130 the top and bottom surfaces of the plate. This could also confirm the inaccuracy of simplifying

131 assumption on full mobilization of shear and normal forces acting on the edges of the plate.

132 Fig. 5 also presents PLA and 3D-FE normalized interaction curves for anchor plate under two-

133 way in-plane translation $\left(H_{x} / H_{x, \max }-H_{y} / H_{y, \max }\right)$ for square and rectangular $(W / L=2)$ plates with

134 thicknesses of $t=L / 20$ and $L / 7$. PLA unconservative tendency to over-estimate the shear bearing

135 capacity is repeated similar to shear-torsion yield envelops. The non-interacting segment appears

136 again at both ends of the yield envelope where maximum shear resistance is expected to diminish

137 due to the shear resistance mobilization in another perpendicular direction according to 3D-FE

138 results. This unconservative trend is more pronounced for plate anchors of smaller aspect ratio

139 and higher thicknesses, as the yield envelope for rectangular plate of $t=L / 20$ nearly tracks 3D-

140 FE data points.

141 Approaching the results of Yang et al. (2010) PLA and 3D-FE yield envelopes and ultimate

142 torsional resistance for smaller thicknesses suggests that Yang's assumption on the negligible

143 interaction of sliding $\left(F_{\mathrm{st}}\right)$ and normal $\left(F_{\mathrm{nt}}\right)$ forces on the edges of the plate at soil failure

144 condition is not accurate for plates of finite thickness as also suggested by Yang et al. (2010). 


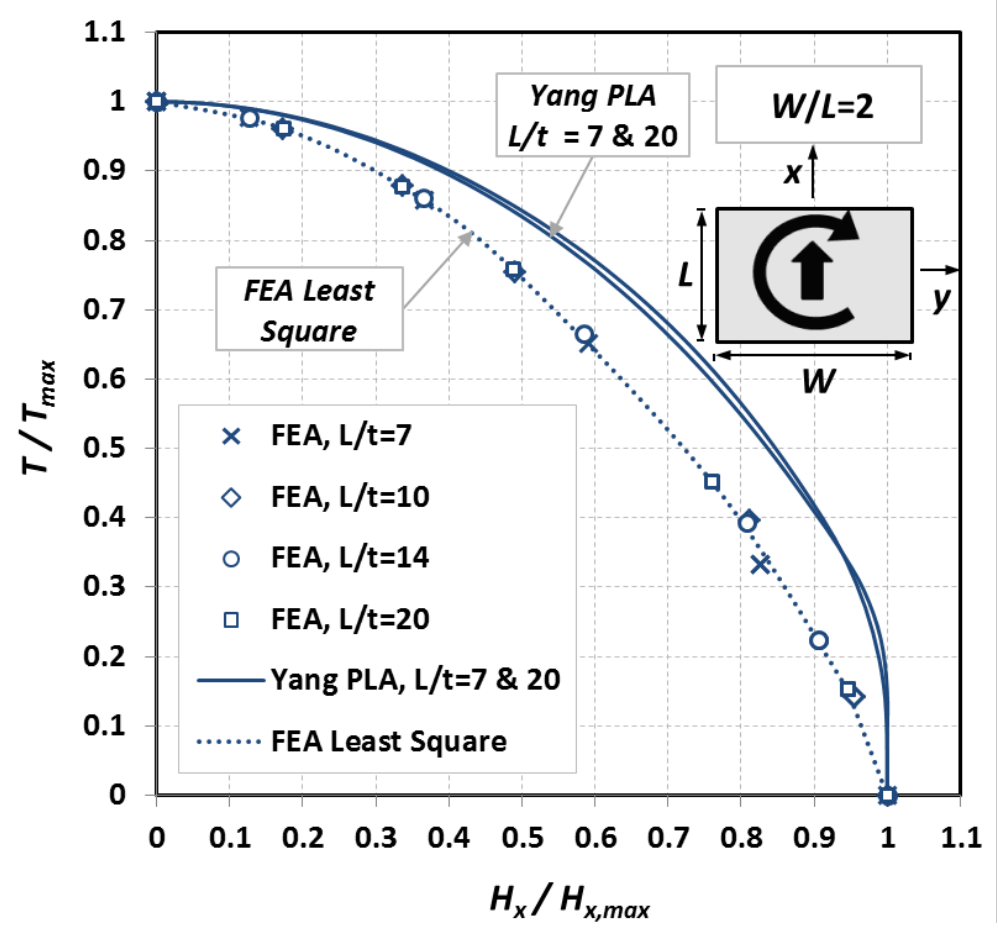

(a)

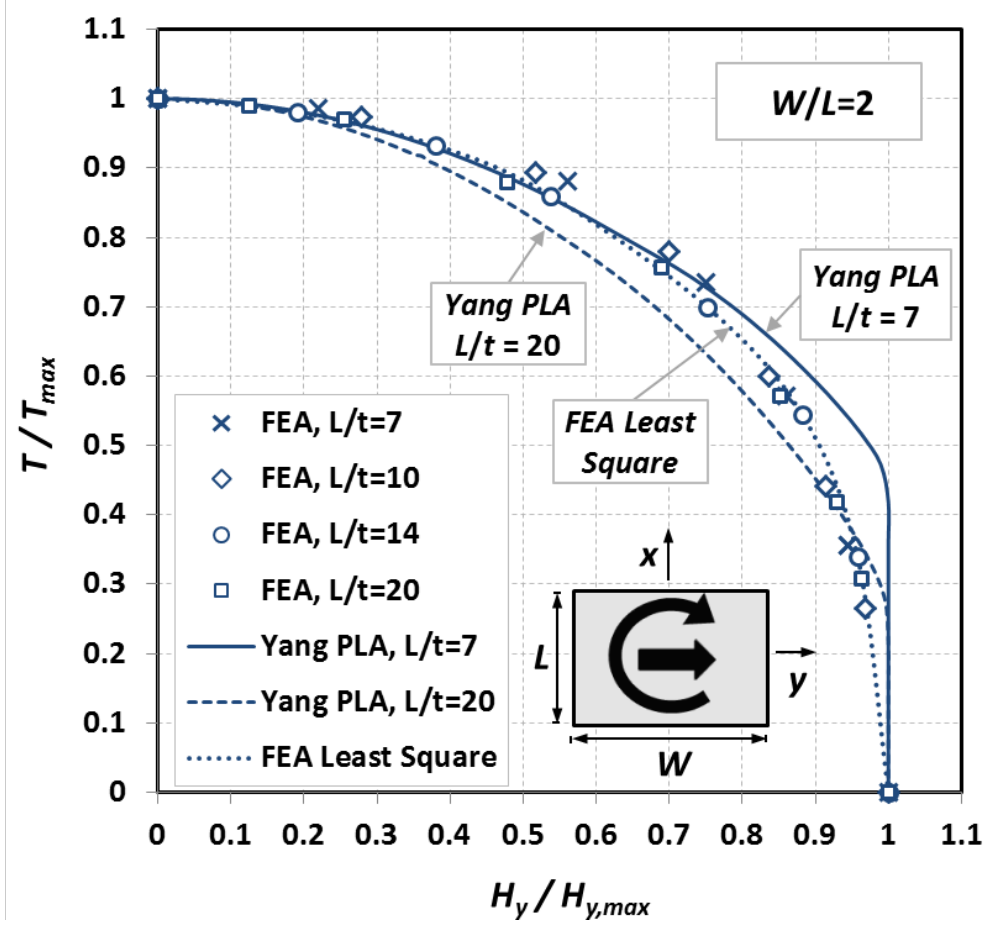

(b)

Figure 3- Comparison of Yang et al. (2010) PLA and 3D-FE predictions for rectangular ( $W / L=2)$ plates under combined: (a) shearx-torsion; (b) sheary-torsion 


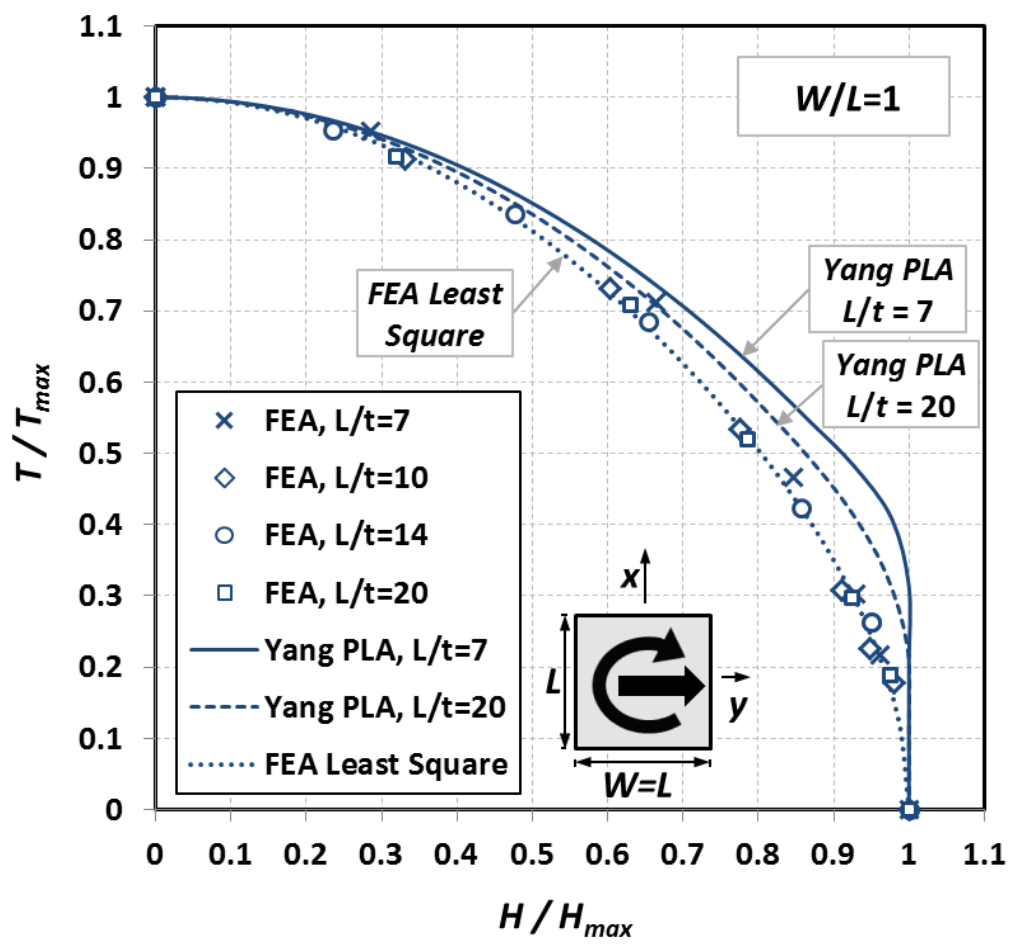

Figure 4- Comparisons of the Yang et al. (2010) PLA and 3D-FE predictions for square $(W / L=1)$ plate under combined shear-torsion

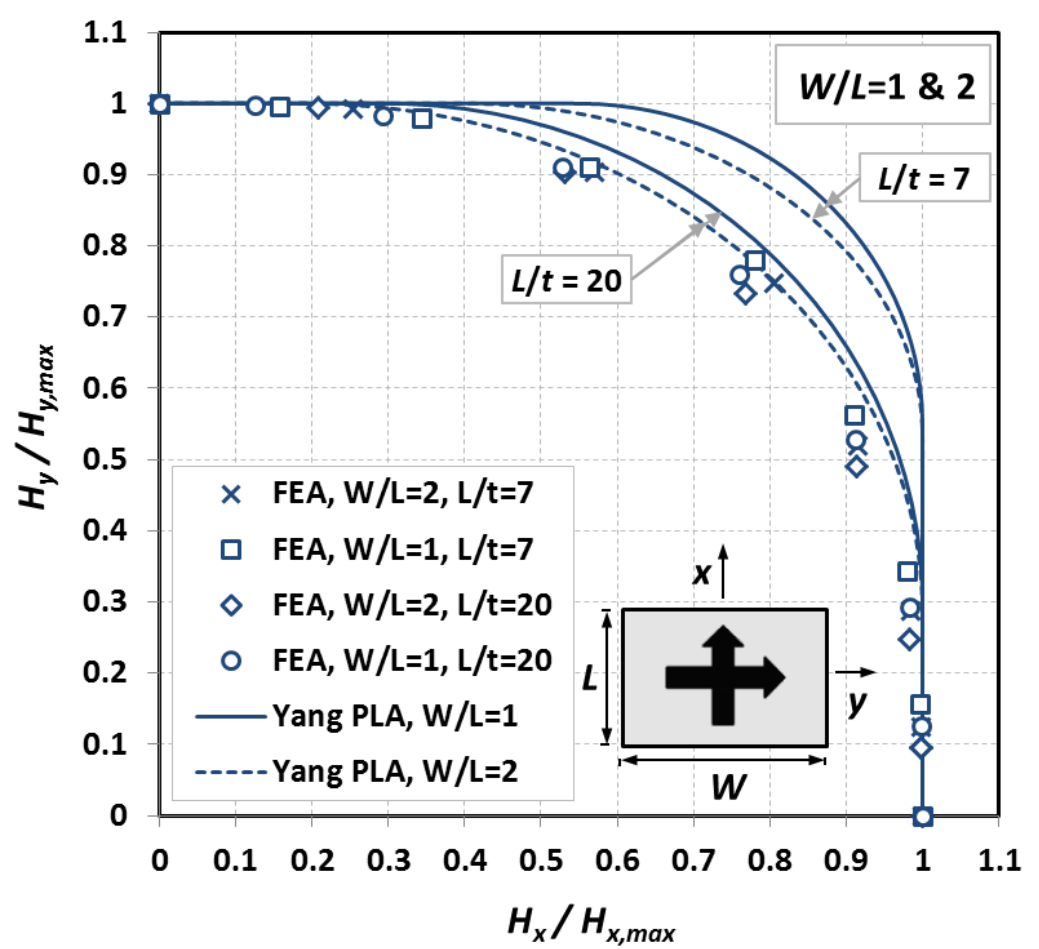

Figure 5- Comparisons of the Yang et al. (2010) PLA and 3D-FE predictions for square and rectangular $(W / L=2)$ plates under coplanar shear $x$ - sheary 
145 As shown in Tables 1 and 2, this assumption could be valid for plates subjected to one-way pure

146 translation where the edges are under pure normal or shear forces, but when the plate is subjected

147 to torsion or combined two-way in-plane translation, there will be a combination of shear and

148 normal reaction forces acting on the plate edges. Neglecting the interaction and assuming full

149 mobilization of both sliding and normal components may result in estimating higher dissipated

150 energy due to soil sliding and normal resistance on the plate edges and over-predicting the

151 overall plate resistance especially for thicker plates. Thus, the insensitivity of 3D-FE derived

152 yield surfaces to plate thickness (see Figs. 3, 4, and 5) is not predicted by the current upper

153 bound solution. Modification of the assumption for interaction of sliding-normal forces on the

154 plate edges could be incorporated in the existing PLA solution to improve the current

155 formulation. The modified PLA solution will be proposed in the following sections of this study.

Table 1. Comparison of PLA and FE results for the ultimate shear force $\left(N_{s, \max }\right)$ and torsion moment $\left(N_{t, \max }\right)$ bearing capacity factors for square plate

\begin{tabular}{|c|c|c|c|c|c|c|}
\hline \multirow{2}{*}{$\begin{array}{l}\text { Mode of } \\
\text { Plate } \\
\text { Loading }\end{array}$} & \multirow{2}{*}{$\begin{array}{c}\text { Plate } \\
\text { thickness } \\
(t / L)\end{array}$} & \multirow{2}{*}{$\begin{array}{c}\text { Finite } \\
\text { Element } \\
\text { Values }\end{array}$} & \multicolumn{2}{|c|}{ Upper Bound Solution } & \multirow{2}{*}{$\begin{array}{l}\text { Difference } \\
\text { of FE and } \\
\text { Yang et al. } \\
(2010)(\%)\end{array}$} & \multirow{2}{*}{$\begin{array}{c}\text { Difference } \\
\text { of FE and } \\
\text { Current } \\
\text { study (\%) }\end{array}$} \\
\hline & & & $\begin{array}{c}\text { Yang et } \\
\text { al. (2010) }\end{array}$ & $\begin{array}{c}\text { Current } \\
\text { (modified) }\end{array}$ & & \\
\hline \multirow{5}{*}{ Shear, $N_{s, \max }$} & 0 & 2 & 2 & 2 & \multicolumn{2}{|c|}{0.0} \\
\hline & $1 / 20$ & 2.90 & 2.85 & 2.85 & \multicolumn{2}{|c|}{-1.7} \\
\hline & $1 / 14$ & 3.30 & 3.21 & 3.21 & \multicolumn{2}{|c|}{-2.7} \\
\hline & $1 / 10$ & 3.67 & 3.70 & 3.70 & \multicolumn{2}{|c|}{0.8} \\
\hline & $1 / 7$ & 4.37 & 4.43 & 4.43 & \multicolumn{2}{|c|}{1.4} \\
\hline \multirow{5}{*}{$\begin{array}{c}\text { Torsion, } \\
N_{t, \max }\end{array}$} & 0 & 0.765 & 0.76 & 0.76 & -0.7 & -0.7 \\
\hline & $1 / 20$ & 1.14 & 1.24 & 1.16 & 8.8 & 1.8 \\
\hline & $1 / 14$ & 1.26 & 1.44 & 1.34 & 14.3 & 6.3 \\
\hline & $1 / 10$ & 1.34 & 1.71 & 1.57 & 27.6 & 17.2 \\
\hline & $1 / 7$ & 1.47 & 2.12 & 1.90 & 44.2 & 29.3 \\
\hline
\end{tabular}


Table 2. Comparison of PLA and FE results for the ultimate shear force $\left(N_{s x, \max }, N_{s y, \max }\right)$ and torsion moment $\left(N_{t, \max }\right)$ bearing capacity factors for rectangular plate $(W / L=2)$

\begin{tabular}{|c|c|c|c|c|c|c|}
\hline \multirow{2}{*}{$\begin{array}{l}\text { Mode of } \\
\text { Plate } \\
\text { Loading }\end{array}$} & \multirow{2}{*}{$\begin{array}{c}\text { Plate } \\
\text { thickness } \\
(t / L)\end{array}$} & \multirow{2}{*}{$\begin{array}{l}\text { Finite } \\
\text { Element } \\
\text { Values }\end{array}$} & \multicolumn{2}{|c|}{ Upper Bound Solution } & \multirow{2}{*}{$\begin{array}{c}\text { Difference } \\
\text { of FE and } \\
\text { Yang et al. } \\
(2010)(\%)\end{array}$} & \multirow{2}{*}{$\begin{array}{c}\text { Difference } \\
\text { of FE and } \\
\text { Current } \\
\text { study } \\
\end{array}$} \\
\hline & & & $\begin{array}{c}\text { Yang et } \\
\text { al. (2010) }\end{array}$ & $\begin{array}{c}\text { Current } \\
\text { (modified) }\end{array}$ & & \\
\hline \multirow{5}{*}{ Shear, $N_{s x, \max }$} & 0 & 2 & 2 & 2 & \multicolumn{2}{|c|}{0.0} \\
\hline & $1 / 20$ & 2.84 & 2.80 & 2.80 & \multicolumn{2}{|c|}{-1.4} \\
\hline & $1 / 14$ & 3.21 & 3.14 & 3.14 & \multicolumn{2}{|c|}{-2.2} \\
\hline & $1 / 10$ & 3.55 & 3.60 & 3.60 & \multicolumn{2}{|c|}{1.4} \\
\hline & $1 / 7$ & 4.20 & 4.29 & 4.29 & \multicolumn{2}{|c|}{2.1} \\
\hline \multirow{5}{*}{ Shear, $N_{s y, \max }$} & 0 & 2 & 2 & 2 & \multicolumn{2}{|c|}{0.0} \\
\hline & $1 / 20$ & 2.50 & 2.48 & 2.48 & \multicolumn{2}{|c|}{-0.8} \\
\hline & $1 / 14$ & 2.72 & 2.68 & 2.68 & \multicolumn{2}{|c|}{-1.5} \\
\hline & $1 / 10$ & 2.93 & 2.95 & 2.95 & \multicolumn{2}{|c|}{0.7} \\
\hline & $1 / 7$ & 3.32 & 3.36 & 3.36 & \multicolumn{2}{|c|}{1.2} \\
\hline \multirow{5}{*}{$\begin{array}{c}\text { Torsion, } \\
N_{t, \max }\end{array}$} & 0 & 1.19 & 1.19 & 1.19 & 0.0 & 0.0 \\
\hline & $1 / 20$ & 1.66 & 1.75 & 1.67 & 5.4 & 0.6 \\
\hline & $1 / 14$ & 1.84 & 2 & 1.89 & 8.7 & 2.7 \\
\hline & $1 / 10$ & 1.98 & 2.32 & 2.18 & 17.2 & 10.1 \\
\hline & $1 / 7$ & 2.27 & 2.81 & 2.59 & 23.8 & 14.1 \\
\hline
\end{tabular}

\section{ANALYTICAL BASELINE SOLUTIONS}

158 This study also introduces a number of convenient analytical and semi-analytical solutions which

159 can provide useful reference solutions to evaluate the modified PLA and FEA approaches.

$160 \quad$ Pure Sliding and Rotation: Limit Equilibrium Approach

161 Nouri et al. (2014) proposed a limit equilibrium (LE) approach to estimate the pure sliding and

162 torsional resistance for shallow foundations. This study uses the same general LE methodology

163 and failure mechanism to derive bearing capacity factors for pure sliding in the $x$ and $y$ directions

164 for a deeply embedded plate anchor of thickness $t$, in a homogeneous soil with an adhesion factor

$165 \alpha$ at the soil-plate interface. Following expressions are developed to calculate bearing capacity 
from the assumed stress distribution and failure mechanism in Fig. 6:

$$
N_{s x, \max }=\frac{H_{\mathrm{x} \max }}{s_{u} L W}=2 \alpha+2\left[\alpha \frac{L}{W}+N_{e}\right] \frac{t}{L}
$$

$$
N_{s y, \max }=\frac{H_{\mathrm{y} \max }}{s_{u} L W}=2 \alpha+2\left[\alpha+N_{e} \frac{L}{W}\right] \frac{t}{L}
$$

where $s_{u}$ is the soil undrained shear strength, $L$ and $W$ are the smaller and larger sides of the

170 plate, and $N_{e}$ is the simple plane strain bearing capacity factor equal to 7.5 (O’Neill et al. 2003).

171 The adhesion factor, $\alpha$, for the fully bonded condition is assumed equal to unity. Note that the

172 results of two and three dimensional sliding resistance are typically normalized by $s_{u} L$ and

$173 s_{u} W L$ respectively. The above expression for pure $x$-shear capacity factor, $N_{s x, \max }$, for a strip plate

$174(L / W \approx 0)$ reduces to $N_{s, \max }=2\left(1+N_{e} . t / L\right)$ as proposed by O'Neill et al. (2003) for ultimate parallel

175 plate capacity in 2D plane strain condition.

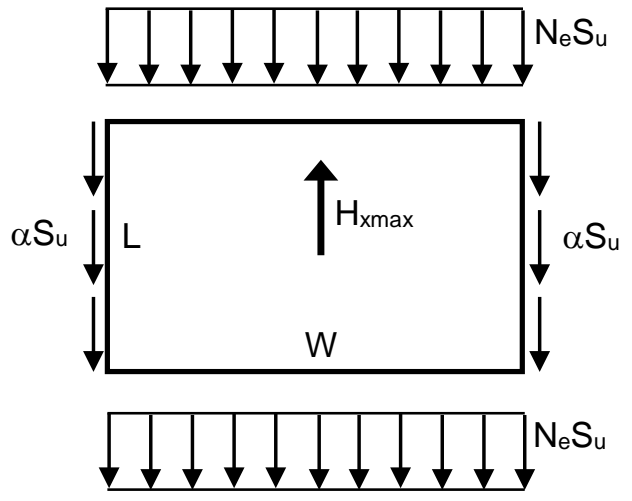

Translation

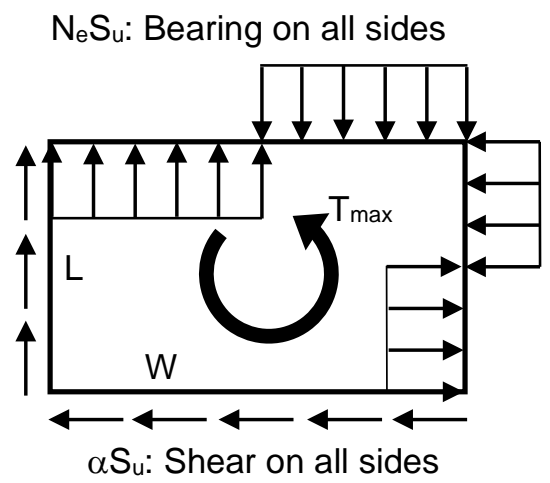

Torsion

Figure 6- Assumed stress distribution of plate pure sliding and torsion for limit equilibrium solution

176 The plate aspect ratio $(W / L)$ does not influence the pure shear capacity of the plate anchor under

$177 x$-sliding, where $N_{\text {sx }}$ (Eq. 2) yields to 4.29 and 4.15 for rectangular $(W / L=2)$ and plane strain strip

178 plate $(W / L=20)$ of $t=L / 7$ (about 3\% different). Evidently, this difference becomes even less for

179 plates of smaller thickness, where in the extreme condition the $N_{\mathrm{sx}}$ becomes independent of $\mathrm{W} / \mathrm{L}$ 
180 for infinitely thin plate ( $N_{\mathrm{sx}}=2$ for square and rectangular plates).

181 For the special case of pure torsion with zero thickness, $t=0$, the following LE solution provides

182 a convenient benchmark for ultimate torsional resistance $T_{\max 0}$ (the subscript ' 0 ' referring to zero

183 thickness) to compare with the numerical calculations:

$184 T_{\max 0}=2 s_{u} \int_{-W / 2}^{W / 2} \int_{-L / 2}^{L / 2} \sqrt{x^{2}+y^{2}} d x d y$

185 Results of the above LE based solution for pure torsion also conforms with Yang et al. (2010)

186 PLA approach where they propose the following closed form solution:

$N_{t \max 0}=T_{\max 0} /\left(s_{u} W L^{2}\right)=$

187

$\alpha \frac{(W / L)^{2}}{6}\left\{\frac{\sin \theta_{o}}{\cos ^{2} \theta_{o}}+\ln \left[\tan \left(\frac{\pi}{4}+\frac{\theta_{o}}{2}\right)\right]\right\}+\alpha \frac{L / W}{6}\left\{\frac{\cos \theta_{o}}{\sin ^{2} \theta_{o}}-\ln \left[\tan \left(\frac{\theta_{o}}{2}\right)\right]\right\}$

188 where $\theta_{o}=\tan ^{-1}(L / W)$. Note that the results of two and three dimensional moment resistance are

189 typically normalized by $s_{u} L^{2}$ and $s_{u} W L^{2}$ respectively.

190 For the general case of a plate with finite thickness, we used a limit equilibrium based approach

191 by summing the torsion resistance on the edges of a rotating plate (Fig. 6). We adopted the

192 simplifying assumption of no interaction effects between bearing (normal) and tangential

193 resistance acting on the plate edges (i.e. full mobilization of these components as shown in Fig.

194 6) which yielded the following expression for additional torsional resistance from plate edges:

195

$$
\Delta N_{t \max e}=C_{f}\left[\frac{1}{2} N_{e}\left(\frac{W}{L}+\frac{L}{W}\right)+2 \alpha\right] \frac{t}{L}
$$

196 A correction factor, $C_{f}$, is included in Eq. 6 to emphasize that some adjustment for interaction

197 effects on plate edges is needed. The total torsional resistance is the sum of Eqs 5 and 6, $N_{\operatorname{tmax} 0}+$

$198 \Delta N_{\text {tmaxe }}$. This closed-form expression portrays the variables affecting torsional capacity and, as

199 will be seen, offers a simple calibration expression to match the finite element solutions. 
201 For plate of zero thickness $(t / L=0)$ an upper bound virtual work analysis provides a useful check

202 for the Yang et al. (2010) PLA and FEA. Nouri et al. (2014) originally introduced this approach

203 to evaluate the resistance of surface foundations (i.e. embedment of zero) under one-way

204 eccentric parallel loading [Fig. 7(b)]. This study generalizes the same approach for a plate of

205 zero thickness subjected to an eccentric translational loading [Fig. 7(a)]: A horizontal load $H$ is

206 applied at a distance $e \operatorname{Cos}(\gamma)$ from the center of the plate, with an eccentricity angle of $\psi$, load

207 angle of $\gamma$, and an associated motion about a center of rotation located a distance $\rho$ from the

208 center. Equating external virtual work, $\dot{W}$, to internal energy dissipation leads to:

$209 \quad H=\frac{\dot{D}}{(\rho+e \operatorname{Cos} \gamma) \dot{\beta}}$

210 where $\dot{\beta}$ is a virtual angular velocity. This relationship is written based on the assumption that

211 the line perpendicular to external force, $H$, through center of rotation is passing through the

212 center of the plate. This assumption has been proved to be valid for infinitely thin plate $(t / L=0)$

213 through PLA parametric analysis. The validity of this assumption could also be evaluated

214 through assuming the center of rotation in a random location and developing the formulation.

215 However, taking advantage of the PLA proved assumption will keep the solution simple which is

216 the main purpose of developing the baseline formulation.

217 The rate of internal energy dissipation $\dot{D}$ is the soil resistance times the local velocity integrated

218 over the plate area:

$219 \dot{D}=2 s_{u} \dot{\beta} \int_{-W / 2}^{W / 2} \int_{-L / 2}^{L / 2} \sqrt{[x+\rho \operatorname{Cos}(\psi+\gamma)]^{2}+[y+\rho \operatorname{Sin}(\psi+\gamma)]^{2}} d x d y$

220 A least upper bound collapse load is obtained by minimizing $H$ in Eq. 7 with respect to $\rho$ and

221 setting it equal to zero, with the assumption of constant $e, \psi$, and $\gamma$ values, which leads to: 
$222 \quad H=\frac{1}{\dot{\beta}} \frac{\partial \dot{D}}{\partial \rho}$

223 For the case of zero plate thickness the collapse load then becomes equal to:

$224 \quad H=2 s_{u} \int_{-W / 2}^{W / 2} \int_{-L / 2}^{L / 2} \frac{\rho_{\text {opt }}+x \operatorname{Cos}(\psi+\gamma)+y \operatorname{Sin}(\psi+\gamma)}{\sqrt{\left[x+\rho_{o p t} \operatorname{Cos}(\psi+\gamma)\right]^{2}+\left[y+\rho_{o p t} \operatorname{Sin}(\psi+\gamma)\right]^{2}}} d x d y$

225 where $\rho_{\text {opt }}$ is the distance to the optimal center of rotation corresponding to a least upper bound.

226 For any arbitrary value of $\rho_{\text {opt }}, \dot{D}$ and $H$ could be derived through numerical evaluation of

227 integrals in Eqs. 8 and 10. The eccentricity, $e$, corresponding to the arbitrary value of $\rho_{o p t}$, is

228 obtained from Eq. 7 with known $\dot{D}, H$, and $\rho_{\text {opt. }}$ Parametric study is also possible with

229 evaluating the equations for a sweep of $\rho_{\text {opt }}$ values.

230 For a special case of eccentricity and loading directions aligned with either the major or minor

231 axis [e.g. eccentricity angle of $\psi=0.0^{\circ}$ and $\gamma=0.0^{\circ}$ as shown in Fig. 7(b)] Eqs. 7, 8, and 10 could

232 be reduced to a more concise form (Nouri et al. 2014):

$233 \quad H=\frac{\dot{D}}{(\rho+e) \dot{\beta}}$

$234 \quad \dot{D}=2 s_{u} \dot{\beta} \int_{-W / 2}^{W / 2} \int_{-L / 2}^{L / 2} \sqrt{(\rho+x)^{2}+y^{2}} d x d y$

$235 \quad H=2 s_{u} \int_{-W / 2}^{W / 2} \int_{-L / 2}^{L / 2} \frac{\rho_{\text {opt }}+x}{\sqrt{\left(\rho_{\text {opt }}+x\right)^{2}+y^{2}}} d x d y$

236 Although the analytical evaluation of the double integral in Eq. 12 to calculate $\dot{D}$ generates a 237 long expression, this equation could be reduced to a single integral (Appendix B) appropriate for 238 the simple design spreadsheet calculations. In addition, analytical integration of Eq. 13 results in 239 a closed-form expression for $H$ : 
$H=2 s_{u}\left[a_{1}^{2} \ln \left|\frac{b_{1}+W / 2}{b_{1}-W / 2}\right|-a_{2}^{2} \ln \left|\frac{b_{2}+W / 2}{b_{2}-W / 2}\right|+W\left(b_{1}-b_{2}\right)\right]$

$a_{1}=\rho_{\text {opt }}+L / 2$

$240 a_{2}=\rho_{\text {opt }}-L / 2$

$b_{1}=\sqrt{a_{1}^{2}+W^{2} / 4}$

$b_{2}=\sqrt{a_{2}^{2}+W^{2} / 4}$

241 Contrary to the PLA approach, the proposed method requires no search or optimization

242 procedure since the final solution directly relates the eccentricity, $e$, to the distance to the optimal

243 center of rotation, $\rho_{\text {opt }}$, and thus the equivalent external force $H=H\left(\rho_{\mathrm{opt}}\right)(\mathrm{Eq} .14)$. This feature

244 also eliminates the complications in optimization procedure in locating the absolute minimum

245 which could be mistaken by the greater local minimums. Regardless of the numerical integration

246 required to evaluate the rate of energy dissipation, the final solution in this method is derived

247 through closed-form expressions easy to implement in spreadsheets. Thus, it can provide a

248 simple robust tool for routine design calculations.

249 Conducting the analysis for a sweep of $\rho$ values generates a predicted reduction in load capacity

$250\left(H / H_{\max }\right)$ versus eccentricity $e$ for a plate under planar eccentric load aligned with the major or

251 minor plate axis. As shown in Fig. 8, shear resistance for loading in the $x$ and $y$ directions show a

252 significant reduction. Square plates, $W / L=1$, experience the greatest decrease in $H$ resistance,

253 with eccentricity as low as $e / L=0.1$ reducing load capacity by more than $5 \%$ and eccentricity $e / L$

$254=0.5$ (load application at the edge of the plate) reducing capacity by more than $40 \%$. With

255 increasing aspect ratio $W / L$ the plate anchor becomes progressively more resistant to eccentric

256 loading. The predictions also show that the reduction in capacity is always the greatest for

257 loading in the $x$ direction; i.e., for load orientations normal to the long axis of the plate. Note that

258 torsion is equal to $T=H$. $e \operatorname{Cos}(\gamma)$. Results of this formulation will be referred as PLA $A_{t=0}$, hereafter. 


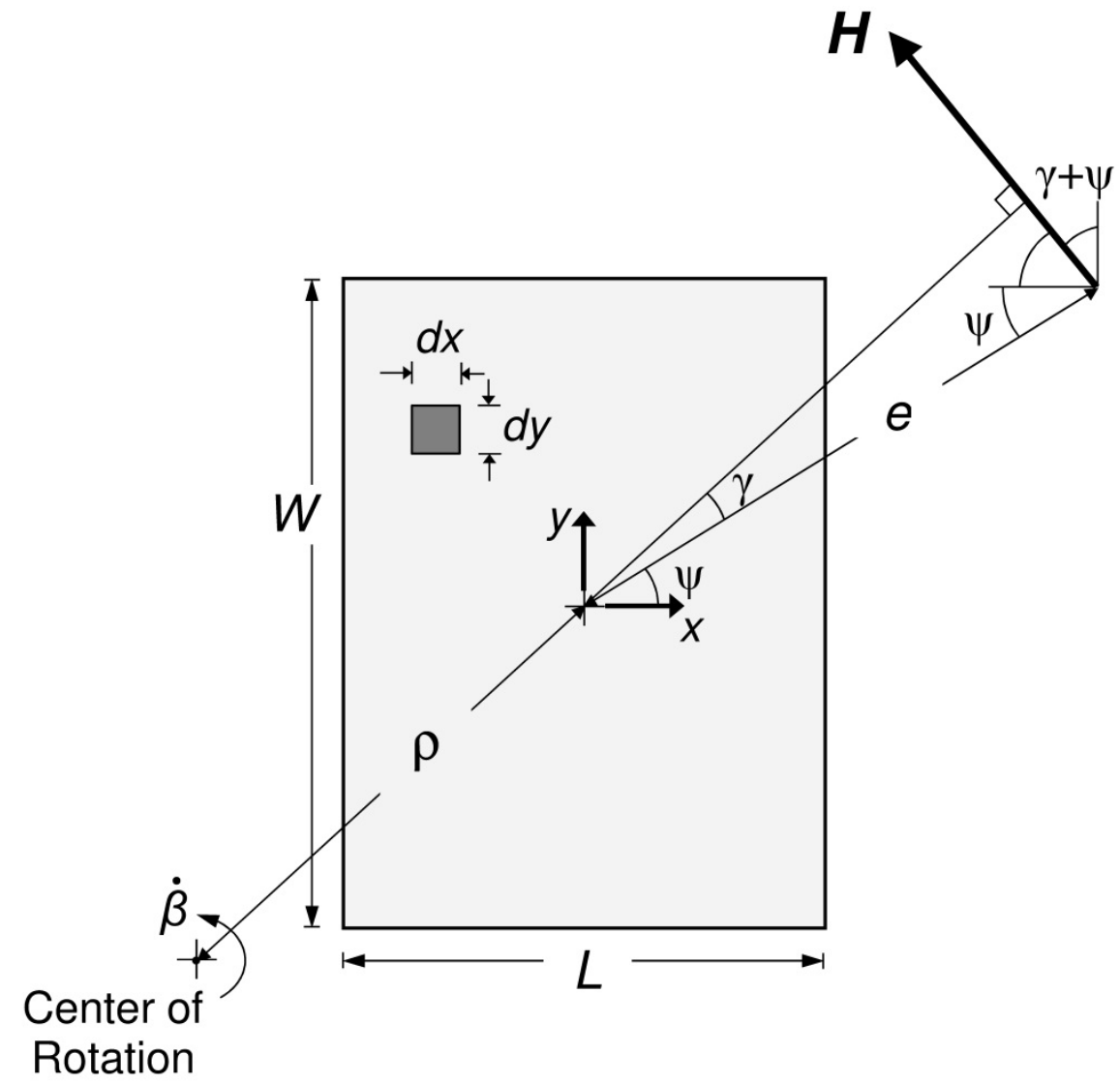

(a)

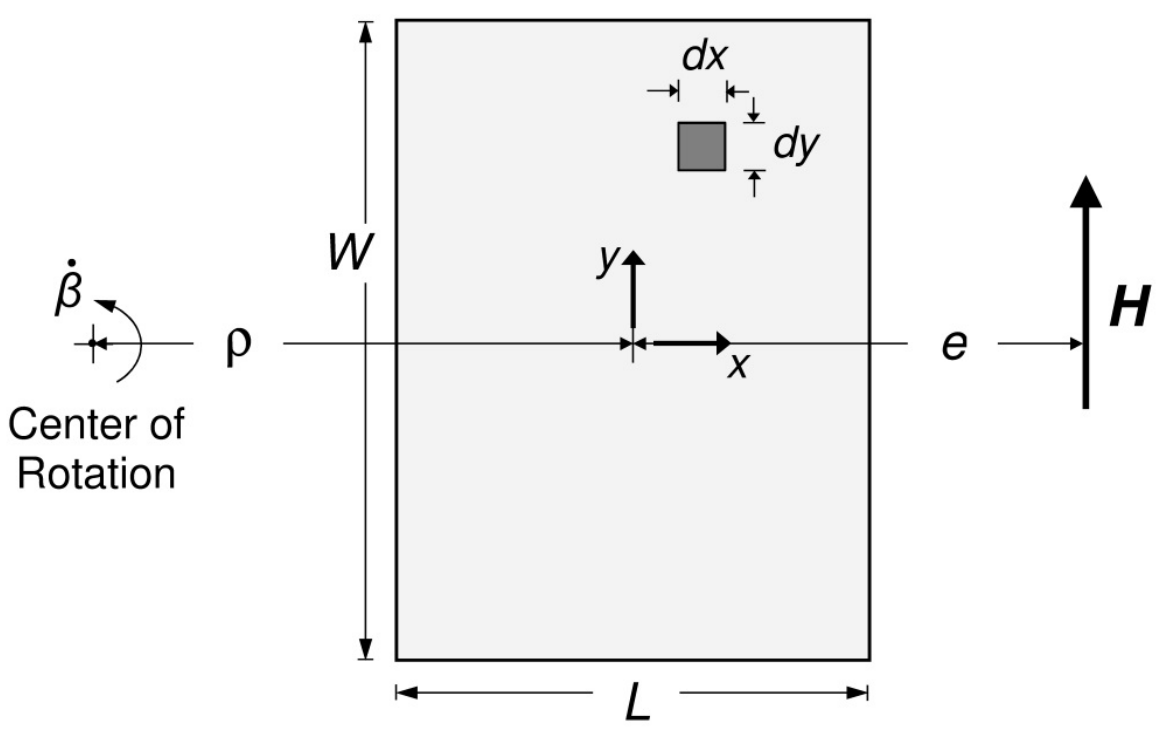

(b)

Figure 7- Model for virtual work analysis for combined sliding-torsion: (a) generalized condition; (b) eccentricity and loading directions aligned with the axis 


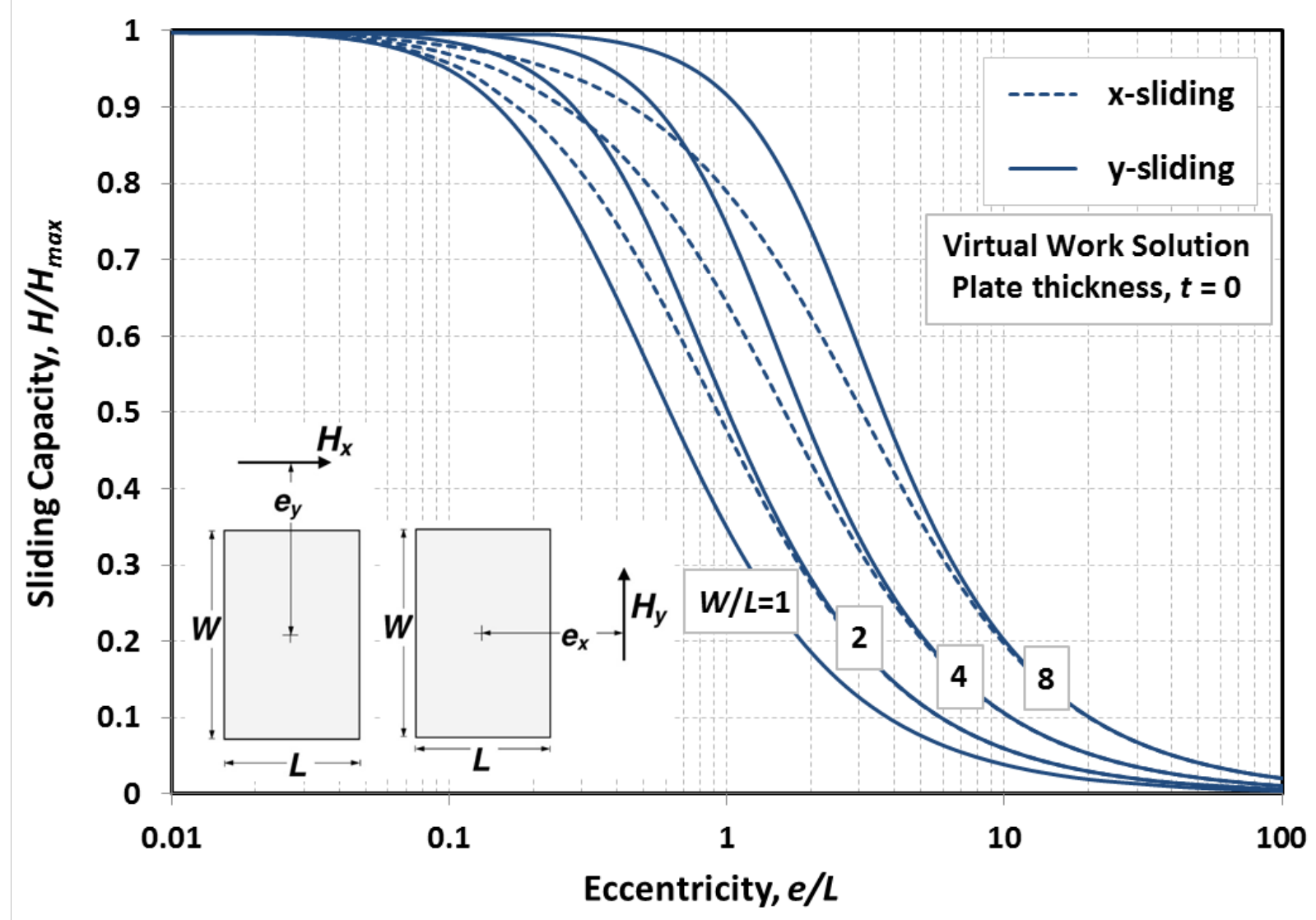

Figure 8- Reduction of shear capacity with eccentricity for square and rectangular plates of zero thickness

\section{Modified PLA solution}

260 To improve the Yang et al. (2010) PLA formulation and incorporate the shear-normal force

261 interaction along the edges of the plate, the dissipation term that accounts for the soil resistance

262 along the plate edges, $\dot{D}_{e}$, should be revised. Other terms of dissipation (i.e. energy dissipation

263 along the top and bottom surfaces of the plate, $\dot{D}_{s}$ ) and external work remains unchanged in the

264 revised formulation.

265 The assumed angular velocity, $\dot{\beta}$, of the plate rotating about point $\mathrm{O}\left(x_{o}, y_{0}\right)$ in Fig. 2, prescribes

266 the relative increments of displacement in the normal and parallel directions with respect to the 267 plate edges as follows (based on the assumption of rigid plate): 
For AB and CD (sides parallel to $x$ axis): $\quad\left\{\begin{array}{l}\dot{u}_{n}=\left|x-x_{0}\right| \dot{\beta} \\ \dot{u}_{s}=\left|y-y_{0}\right| \dot{\beta}\end{array}\right.$

For AD and BC (sides parallel to $y$ axis): $\quad\left\{\begin{array}{l}\dot{u}_{n}=\left|y-y_{0}\right| \dot{\beta} \\ \dot{u}_{s}=\left|x-x_{0}\right| \dot{\beta}\end{array}\right.$

268 where $\dot{u}_{n}$ and $\dot{u}_{s}$ are displacement increments normal and parallel to the plate vertical end faces,

269 respectively. A general relationship for the sliding-normal interaction curve, $f\left(N_{n t}, N_{s t}\right)$, is 270 adopted as follows:

$$
f\left(N_{n t}, N_{s t}\right)=\left(\frac{N_{n t}}{N_{n t, \text { max }}}\right)^{m}+\left(\frac{N_{s t}}{N_{s t, \text { max }}}\right)^{n}-1=0
$$

271 where $N_{n t}=F_{n t} /\left(s_{u} \times t \times d l\right)$ and $N_{s t}=F_{s t} /\left(s_{u} \times t \times d l\right)$ are non-dimensional forms of $F_{n t}$ and $F_{s t}$

272 (respectively normal and shear forces on the plate edges in Fig. 2) acting on arbitrary element of 273 dimensions $t$ (plate thickness) by $d l(=d x$ and $d y$ for sides parallel to $x$ and $y$ axis respectively) on

274 the plate edges as shown in Fig. 2. $N_{n t, \max }=N_{e}$ which is the plane strain bearing capacity factor, is 275 typically equal to 7.5 (O’Neill et al. 2003). The maximum sliding force $N_{s t, \max }$ is equal to 276 adhesion factor $\alpha$ and assumed to be unity for the plate fully bonded to the soil. The assumption 277 of no detachment is necessary for deriving a limit analysis solution with an associated flow rule, 278 but the adhesion factor could take any value between zero and unity. Here the assumption of $\alpha=1$ 279 is consistent with the fully bonded condition. The center of area of the element on the edge of the 280 plate with coordinates $(x, y)$ is assumed to be the representative point of the element. As 281 discussed by Prager (1959) the loads and plastic displacements associated with yielding of the 282 foundation are treated as generalized plastic strains. As the upper bound solution is obtained for 283 failure conditions, this study assumes that the increments of elastic displacement are negligible 284 when compared to the plastic increments. Therefore, assuming an associated flow rule, 285 increments of total displacements of the anchor plate in directions normal and tangential to the 
286 plate edges at point $(x, y)$ at the failure condition could be calculated as follows:

$$
\begin{aligned}
& \dot{u}_{n t}=\dot{u}_{n t}^{p}=\lambda \frac{\partial f\left(N_{n t}, N_{s t}\right)}{\partial N_{n t}}=\lambda \frac{m N_{n t}^{m-1}}{\left(N_{n t, \max }\right)^{m}} \\
& \dot{u}_{s t}=\dot{u}_{s t}^{p}=\lambda \frac{\partial f\left(N_{n t}, N_{s t}\right)}{\partial N_{s t}}=\lambda \frac{n N_{s t}^{n-1}}{\left(N_{s t, \max }\right)^{n}}
\end{aligned}
$$

287 By dividing the above two expressions and substituting $\dot{u}_{n}$ and $\dot{u}_{s}$ from Eqs. (15-a) and (15-b)

288 for each plate edge, $\dot{u}_{n t}, \dot{u}_{s t}, \lambda$ and $\dot{\beta}$ are eliminated and expression in terms of $N_{n t}$ and $N_{s t}$ are 289 obtained as follows:

For $\mathrm{AB}$ and $\mathrm{CD}$ (sides

parallel to $x$ axis):

$$
\frac{\left|x-x_{0}\right|}{\left|y-y_{0}\right|}=\left(\frac{m}{n}\right)\left(\frac{N_{n t}^{m-1}}{N_{s t}^{n-1}}\right)\left(\frac{N_{s t, \max }^{n}}{N_{n t, \text { max }}^{m}}\right)
$$

For $\mathrm{AD}$ and $\mathrm{BC}$ (sides

parallel to $y$ axis):

$$
\frac{\left|y-y_{0}\right|}{\left|x-x_{0}\right|}=\left(\frac{m}{n}\right)\left(\frac{N_{n t}^{m-1}}{N_{s t}^{n-1}}\right)\left(\frac{N_{s t, \text { max }}^{n}}{N_{n t, \text { max }}^{m}}\right)
$$

290 Going through the details to implement the sliding-normal force interaction relationship in the 291 plasticity solution indicates that in order to obtain a "closed from" of the upper bound solution, $m$ 292 should be equal to $n(m=n)$. For simplicity a circular relationship for the sliding-normal 293 interaction curve is adopted $(m=n=2)$ :

For AB and CD (sides parallel to $x$ axis): $\quad \frac{\left|x-x_{0}\right|}{\left|y-y_{0}\right|}=\left(\frac{N_{n t}}{N_{s t}}\right)\left(\frac{N_{s t, \max }}{N_{n t, \max }}\right)^{2}$

For AD and BC (sides parallel to $y$ axis): $\quad \frac{\left|y-y_{0}\right|}{\left|x-x_{0}\right|}=\left(\frac{N_{n t}}{N_{s t}}\right)\left(\frac{N_{s t, \max }}{N_{n t, \max }}\right)^{2}$

294 Therefore, having two equations, (19-a) or (19-b) and the yield surface, Eq. (16), the two 295 unknowns, $N_{n t}$ and $N_{s t}$, could be determined: 
For AB and CD (sides
parallel to $x$ axis): $\left\{\begin{array}{l}N_{s t}=N_{s t, \text { max }}\left[\left(\frac{N_{n t, \text { max }}}{N_{s t, \text { max }}} \cdot \frac{\left|x-x_{0}\right|}{\left|y-y_{0}\right|}\right)^{2}+1\right]^{-0.5} \\ N_{n t}=\left(\frac{N_{n t, \text { max }}}{N_{s t, \text { max }}}\right)^{2}\left(\frac{\left|x-x_{0}\right|}{\left|y-y_{0}\right|}\right) N_{s t}\end{array}\right.$

For AD and BC (sides
parallel to $y$ axis): $\left\{\begin{array}{l}N_{s t}=N_{s t, \text { max }}\left[\left(\frac{N_{n t, \text { max }}}{N_{s t, \text { max }}} \cdot \frac{\left|y-y_{0}\right|}{\left|x-x_{0}\right|}\right)^{2}+1\right]^{-0.5} \\ N_{n t}=\left(\frac{N_{n t, \text { max }}}{N_{s t, \text { max }}}\right)^{2}\left(\frac{\left|y-y_{0}\right|}{\left|x-x_{0}\right|}\right) N_{s t}\end{array}\right.$

296 Having $N_{n t}$ and $N_{s t}$ in terms of element position $(x, y)$ and point of rotation $\left(x_{0}, y_{0}\right)$, the increment 297 of energy dissipation rate, e.g. for a representative element on BC, could be obtained as follows:

$$
d \dot{D}_{e, B C}=\left(F_{s t}\left|x-x_{0}\right|+F_{n t}\left|y-y_{0}\right|\right) \dot{\beta} d y=t s_{u}\left(N_{s t}\left|x-x_{0}\right|+N_{n t}\left|y-y_{0}\right|\right) \dot{\beta} d y
$$

298 Where $x$ is constant for side BC of the plate, i.e. $x=L / 2$. Therefore, the total rate of dissipated 299 energy along the BC side is calculated by integrating the above expression:

$$
\dot{D}_{e, B C}=\int_{-W / 2}^{W / 2} d \dot{D}_{e, B C}=\int_{-W / 2}^{W / 2} s_{u} \dot{\beta}\left(N_{s t}\left|\frac{L}{2}-x_{0}\right|+N_{n t}\left|y-y_{0}\right|\right) t d y
$$

300 Following the same procedure, the rate of energy dissipation for the other edges of the plate can 301 be derived:

$$
\begin{aligned}
& \dot{D}_{e, A B}=\int_{-L / 2}^{L / 2} s_{u} \dot{\beta}\left(N_{s t}\left|\frac{W}{2}-y_{0}\right|+N_{n t}\left|x-x_{0}\right|\right) t d x \\
& \dot{D}_{e, C D}=\int_{-L / 2}^{L / 2} s_{u} \dot{\beta}\left(N_{s t}\left|\frac{W}{2}+y_{0}\right|+N_{n t}\left|x-x_{0}\right|\right) t d x \\
& \dot{D}_{e, A D}=\int_{-W / 2}^{W / 2} s_{u} \dot{\beta}\left(N_{s t}\left|\frac{L}{2}+x_{0}\right|+N_{n t}\left|y-y_{0}\right|\right) t d y
\end{aligned}
$$

302 Where $N_{\text {st }}$ and $N_{n t}$ are substituted from Eqs. (20-a) and (20-b). Thus, the rate of energy 
303 dissipation due to soil resistance on the plate edges is determined:

$$
\dot{D}_{e}=\dot{D}_{e, A B}+\dot{D}_{e, C D}+\dot{D}_{e, B C}+\dot{D}_{e, A D}
$$

304 The other terms of dissipation and external work remain unchanged in the revised formulation.

305 The basis of the Yang and modified PLA solutions are summarized in Appendix A.

\section{COMPARISON TO BASELINE AND PLA SOLUTIONS}

308 The finite element studies are now compared to the baseline and PLA solutions through

309 comparing the two basic components defining the yield envelope:

310 1- The "size” of the yield envelope quantified by the predicted maximum resistance under 311 pure translational $\left(H_{x, \max }, H_{y, \max }\right)$ and rotational $\left(T_{\max }\right)$ loading.

$312 \quad 2-\quad$ The "shape" of the yield envelope defined by the mathematical model $f\left(H_{x} / H_{x, \max }, H_{y}\right.$ $\left./ H_{y, \max }, T / T_{\max }\right)=0$ which is curve fitted to the yield surface, and represented by interaction factors (i.e. $h x, h y, h$, and $m z$ ):

$$
f\left(\frac{H_{x}}{H_{x, \max }}, \frac{H_{y}}{H_{y, \max }}, \frac{T}{T_{\max }}\right)=\left[\left(\frac{H_{x}}{H_{x, \max }}\right)^{h x}+\left(\frac{H_{y}}{H_{y, \max }}\right)^{h y}\right]^{h}+\left(\frac{T}{T_{\max }}\right)^{m z}-1=0
$$

315 The mathematical relationship in Eq. 25 represents the ellipsoid interaction or yield surface in

316 the $H_{x} / H_{x, \max }-H_{y} / H_{y, \max }-T / T_{\max }$ normalized space.

\section{Size of the Yield Envelope: Pure Translation and Rotation}

318 FEA, LE, and modified PLA shear and torsional bearing capacity factors are compared in Fig. 9.

319 The FEA maximum shear resistance, $N_{s, x \max }$ and $N_{s, y \max }$, increases linearly with increasing plate

320 thickness, $t$, similar to predictions from LE (Eqs. 2 and 3). FEA shear bearing factors are also in

321 good agreement with LE predictions. Although the LE solutions increasingly over-predict the

322 FEA values with increasing plate thickness, the difference does not exceed $2.8 \%$ which occurs 
323 for the thickest square plate $(t / L=1 / 7)$. Note that $L E$ and PLA methods generate the same

324 solutions for the ultimate shear capacity.

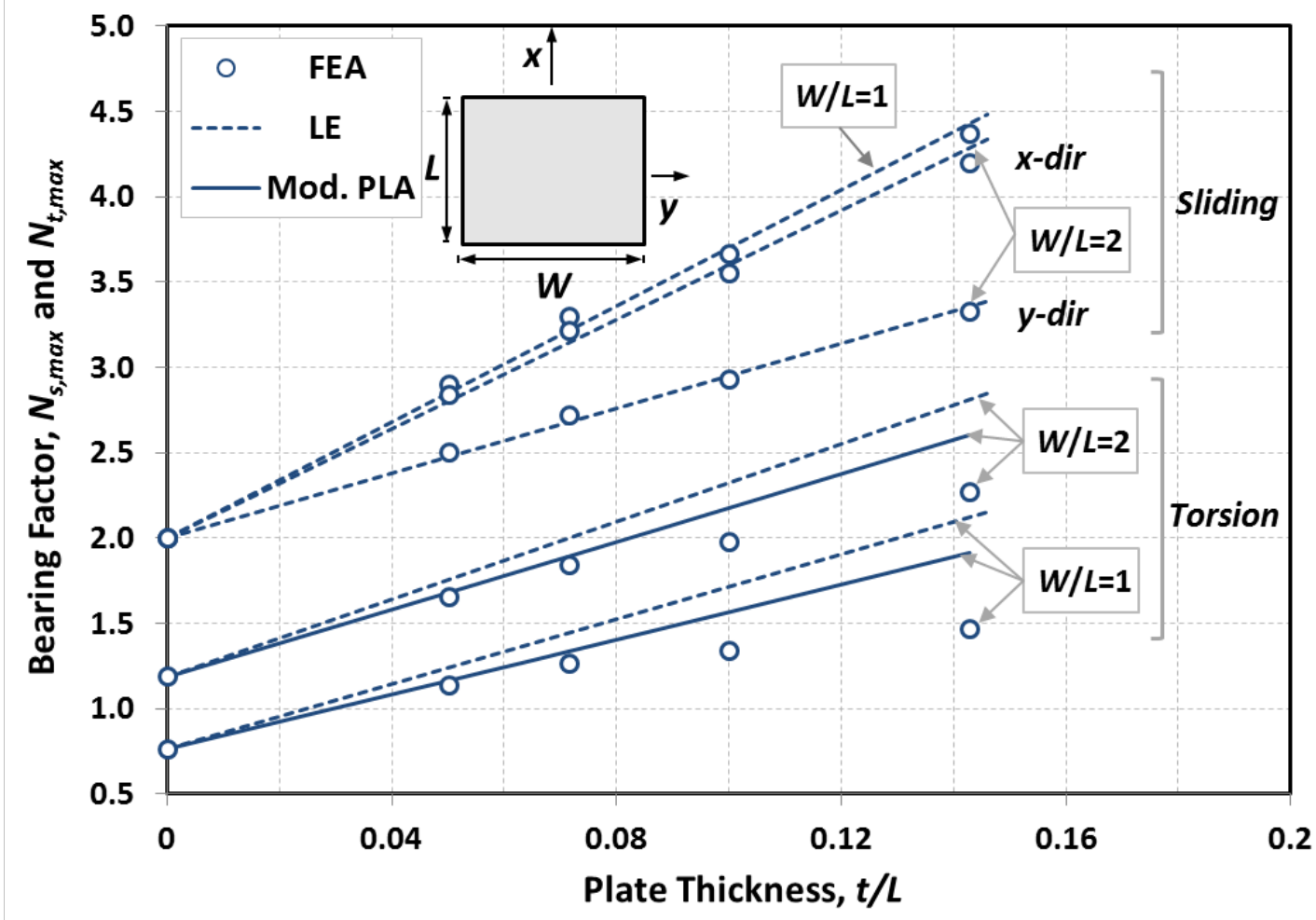

Figure 9- Normalized maximum resistance for pure translation and torsion

325 As for the torsional resistance, $N_{t, \max }$, the FEA solution does not show a linear trend predicted by 326 both the LE (Eqs. 5 and 6) and modified PLA formulations (Table 1 and 2; Fig. 9). With plate

327 thickness increasing, the uncorrected $\left(C_{f}=1\right)$ LE values gradually exceed the FEA solution, by 328 up to $44 \%$ for square plate of $t / L=1 / 7$. It can be shown that an interaction factor $C_{f}=0.67 \mathrm{in}$ Eq.

3296 will make the limit equilibrium predictions a good fit for the finite element solutions for both

330 the square and rectangular plates. Likewise, modified PLA tends to overestimate the increase in

331 torsional capacity due to plate thickness. However, taking into account the interaction effect of

332 the normal and tangential forces acting on the plate edges brings the modified PLA predictions

333 closer to FEA values compared to Yang et al. (2010) PLA as compared in Tables 1 and 2 (e.g. 
maximum difference of $29 \%$ compared to $44 \%$ for square plate of $t / L=1 / 7)$. Over-estimation of

335 the capacity by the modified PLA with increasing thickness is also expected since the PLA

336 formulation is based on "upper bound” limit analysis solution.

\section{Shape of Yield Envelope: Combined Loading}

338 Figs. 10 and 11 compare the FEA shear-torsion interaction envelopes to modified PLA and the

339 PLA virtual work solution for infinitely thin plate (i.e. PLA $t=0$, Eqs. 7-14). Load capacities are

340 presented in normalized form, $H / H_{\max }$ versus $T / T_{\max }$, for rectangular (Fig. 10) and square plates

341 (Fig. 11). All the FEA predictions for the plate thickness range of $t=L / 7$ to $L / 20$ are essentially

342 lying on a single curve indicating that the shape of the yield envelopes is independent of the plate

343 thickness. A noticeable improvement is observed in modified PLA predictions for interaction

344 response (Figs. 10 and 11) when compared with the Yang et al. (2010) PLA predictions (Figs. 3-

345 5). Although the modified PLA values are slightly unconservative especially for thicker plates,

346 the modified PLA predictions are generally less sensitive to the plate thickness compared to

347 Yang et al. (2010) approach and also in overall good agreement with the FEA solutions.

348 The insensitivity of the FEA interaction response with respect to variations in plate thickness and

349 close agreement to the FEA yield envelopes, introduces the PLA $\mathrm{P}_{t=0}$ solution as a realistic

350 portrayal of the yield envelope for non-zero thickness (Fig. 11). However, except for the

351 rectangular plate under $x$-shear and torsion, the $\mathrm{PLA}_{t=0}$ predictions tend to be slightly on a

352 conservative side.

353 Fig. 12 shows the modified PLA and FEA interaction diagrams for the plate under co-planar

354 translation (i.e. non-eccentric loading with varying load directions $\gamma$ ). With some tendency to be

355 on an unconservative side, especially for thicker plates, the modified PLA predictions are in

356 good agreement with the FEA solutions. The minimal influence of the plate thickness on the 
357 shape of FEA derived yield envelopes is better captured by the modified PLA solutions

358 compared to the Yang PLA approach (Fig. 5). In general, the modified PLA offers markedly

359 improved predictions compared to Yang et al. (2010) PLA (Fig. 5) for plate under co-planar

360 shear. For the case of zero thickness, the yield envelope obtained from the PLA $A_{t=0}$ solution, will

361 be circular for both square and rectangular plates. The theoretical basis is easy to explain: for

362 plate with area of base $=A$, thickness $=$ zero, and soil undrained shear strength $=s_{u}$ the sliding

363 resistance will be constant and equal to $H_{\max }=2 A s_{u}$, regardless of the direction of sliding for

364 Tresca yield criterion. This value actually represents the radius of the circular yield locus in the

$365 H_{x}-H_{y}$ space as shown in Fig. 12. This circular interaction relationship in $H_{x}-H_{y}$ space applies

366 for a theoretically infinitely thin plate regardless of the shape of geometric base. Fig. 12 indicates

367 that the plate thickness induces a small but noticeable departure from a circular envelope in both

368 PLA and FEA solutions. This departure is greater for thicker plates with lower aspect ratio and

369 more pronounced in modified PLA predictions compared to FEA results. A simplifying

370 assumption of a circular $H_{x} / H_{x, \max }-H_{y} / H_{y, \max }$ yield envelope (i.e. $h x=h y=2$ ) therefore generates

371 reasonably realistic predictions, albeit underestimating the FEA yield envelope by about $13 \%$.

372 Adopting interaction coefficients of $h x=h y=2.5$ in Eq. 25 generates a more realistic yield

373 envelope which tracks the FEA derived solutions. 


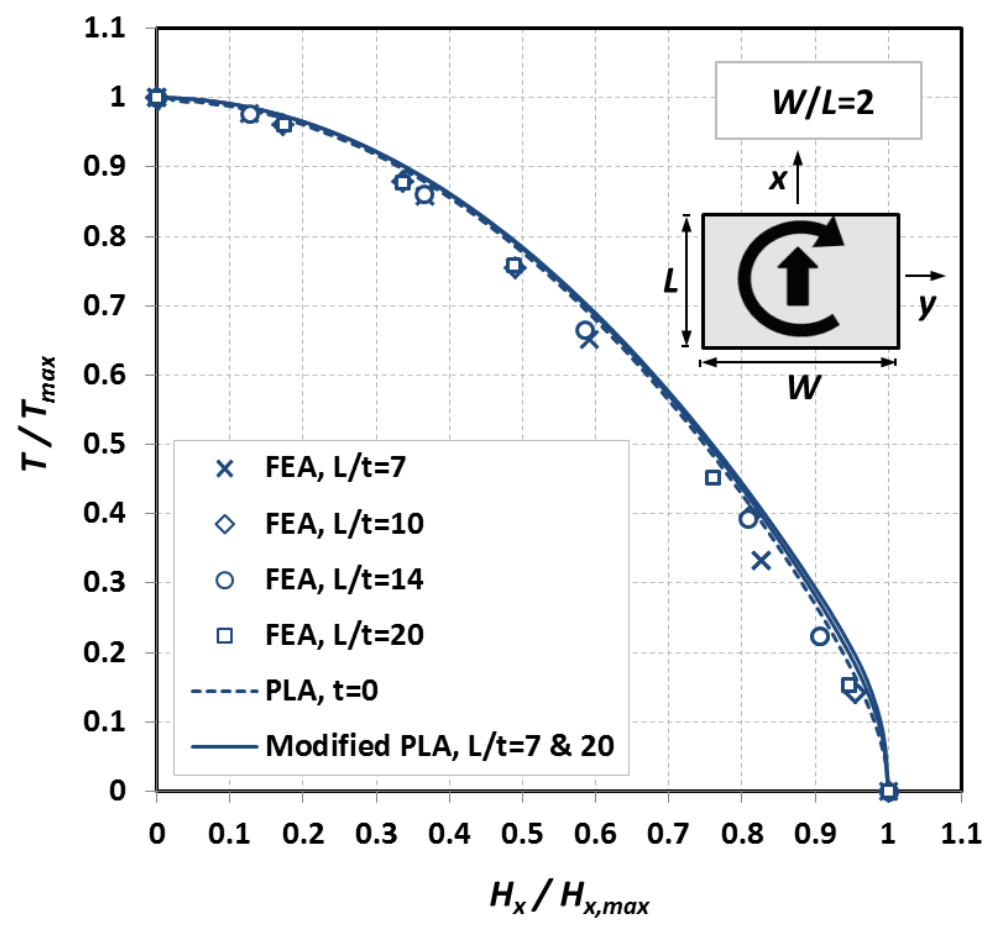

(a)

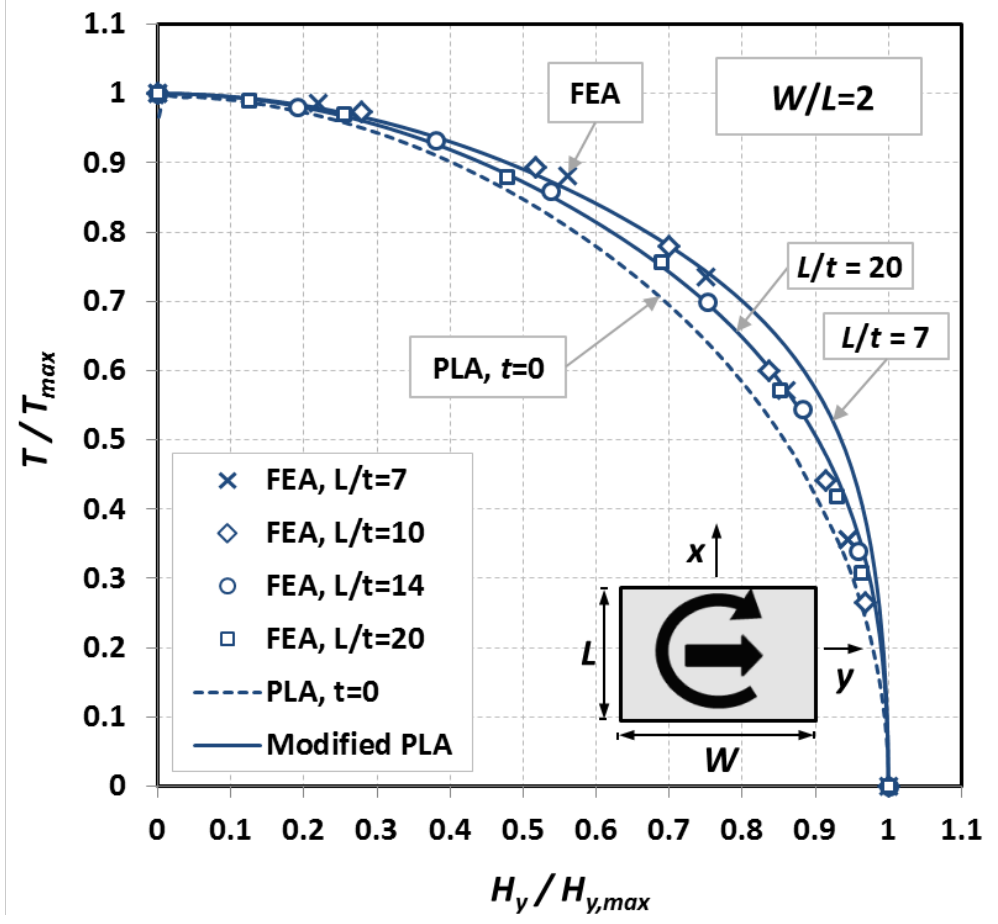

(b)

Figure 10- Analytical and FEA yield envelopes for rectangular plate: (a) shearx-torsion interaction; (b) sheary-torsion interaction 


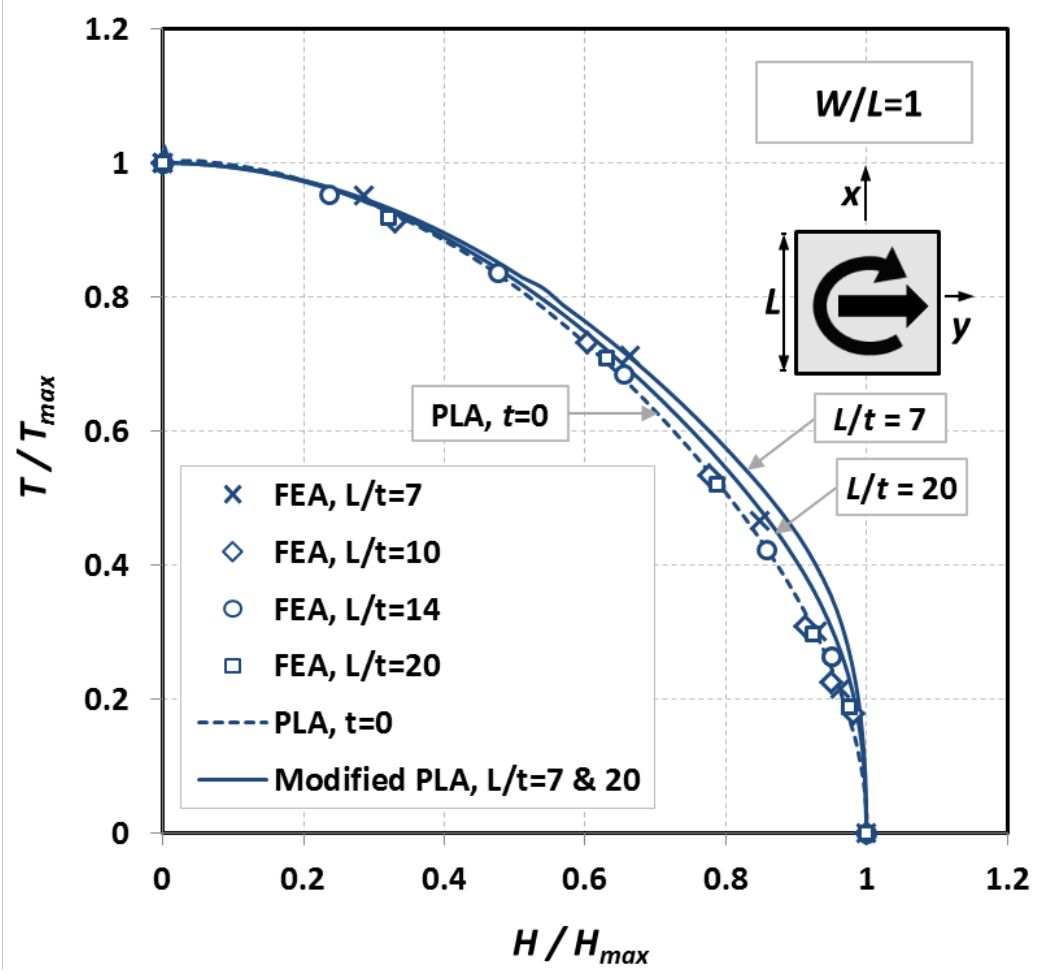

Figure 11- Analytical and FEA yield envelopes for shear-torsion interaction in square plate

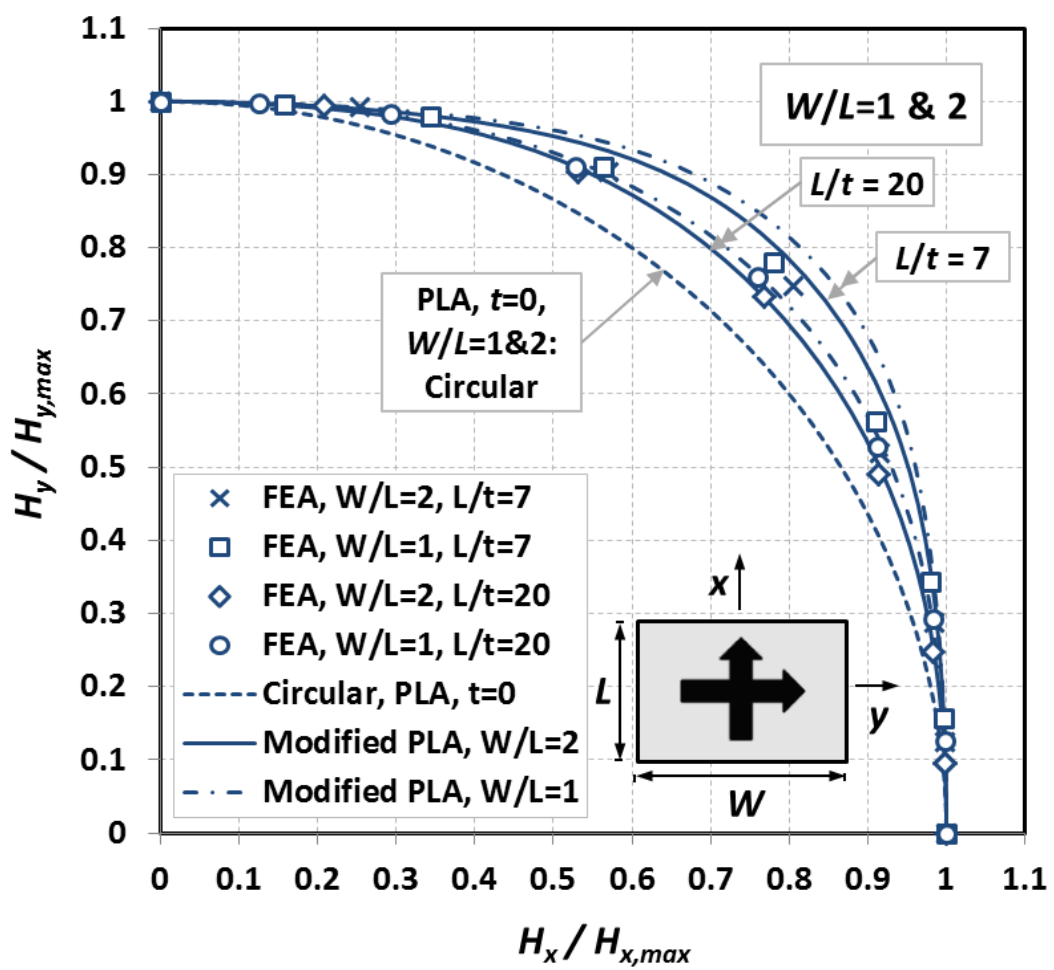

Figure 12- Analytical and FEA yield envelopes for shearx-sheary, square and rectangular plates 


\section{ECCENTRICITY AND LOAD CAPACITY REDUCTION}

375 One-directional shear-torsion $\left(\mathrm{H}_{x}-\mathrm{T}\right.$ and $\left.\mathrm{H}_{y}-\mathrm{T}\right)$ and zero load angle $(\gamma=0)$

376 The reduction effect of torsion on plate sliding capacity and the combined loading effects could 377 also be portrayed through the effect of eccentricity on the load capacity. Figs. 13-16 illustrate the 378 sliding load reduction versus the eccentricity for square and rectangular plates of $W / L=2,4$, and

3798 with thickness of $t=L / 7$. Both sliding load capacity and eccentricity are expressed in

380 dimensionless form of $N=H / s_{u} L W$ and $e / L$, respectively. The sliding load factor $\left(N=H / s_{u} L W\right)$ is

381 not normalized by the maximum pure capacity in Figs. 13-16, which makes the capacity

382 reduction curves convenient to estimate both for the magnitude of shear resistance as well as the

383 reduction/interaction effect. As discussed previously, the insensitivity of the shape of FEA

384 sliding-torsion yield envelope and fairly accurate and simple baseline solutions to predict size

385 and shape of the yield locus, provides the opportunity to develop a simplified approach to predict

386 the shear-torsion plate capacity. The PLA virtual work baseline solution for zero thickness

$387 \quad\left(\mathrm{PLA}_{t=0}\right)$ plate under combined loading (Fig. 8) was adopted in combination with the limit

388 equilibrium derived equations for pure translational load capacity ( $H_{\max }$ using Eqs. 2 and 3 which

389 proved to be fairly accurate), to generate the simplified solution in Figs. 13-16.

390 The FEA predictions for the square and rectangular $(W / L=2)$ plates are compared with the

391 simplified and modified PLA solutions in Figs. 13 and 14, while Figs. 15 and 16 just include the

392 results of analytical simplified and modified PLA predictions for plates of $W / L=4$ and 8 . Figs.

393 14-16 also evaluate the effect of eccentricity angle $(\psi)$ for the rectangular plates sliding in $x$ and

$394 y$ directions $\left(\psi=90^{\circ}\right.$ and $0^{\circ}$ when $\left.\gamma=0^{\circ}\right)$ with various eccentricities. 


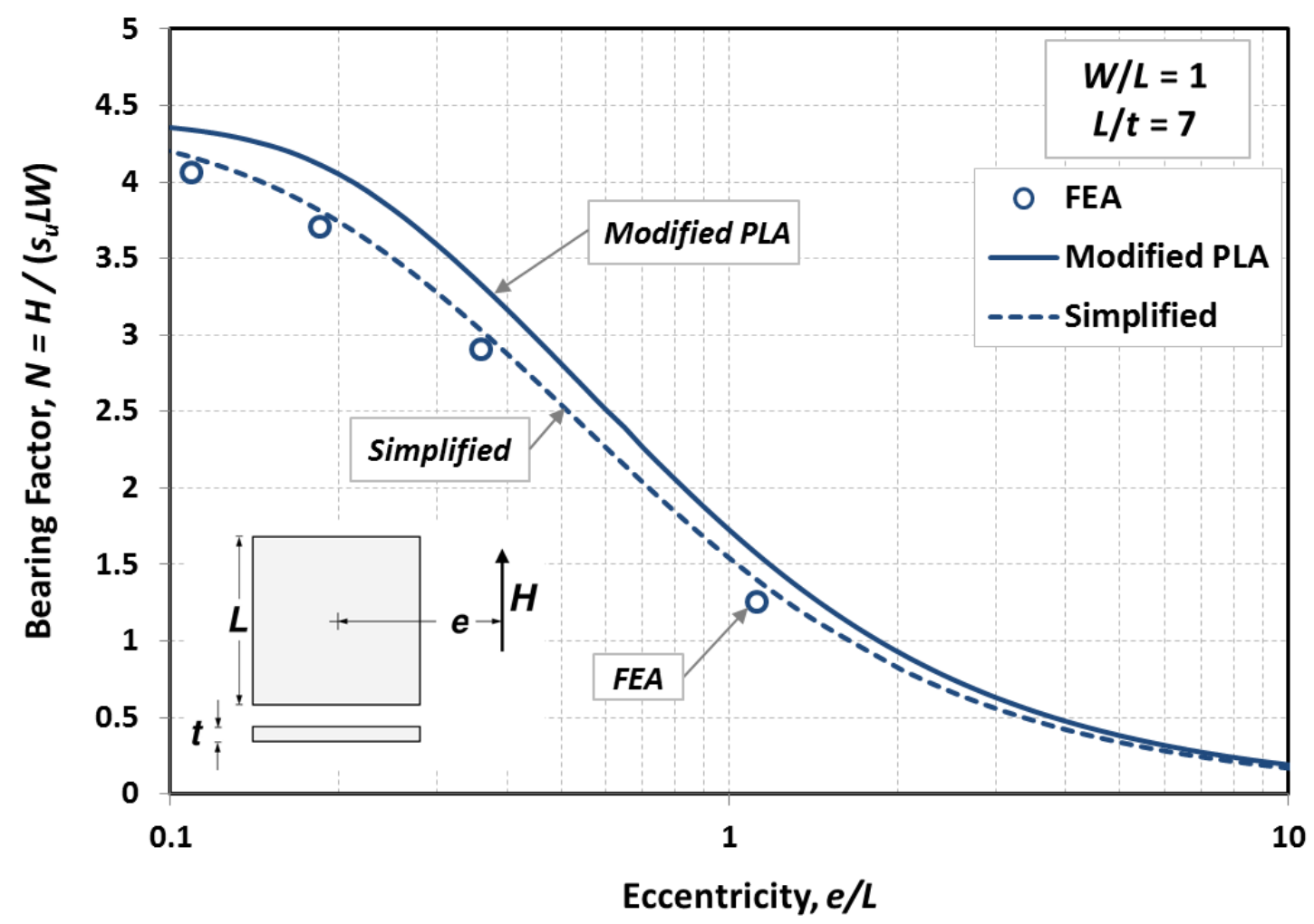

Figure 13- Reduction effect of eccentricity on sliding load capacity for square plate of $t=L / 7$

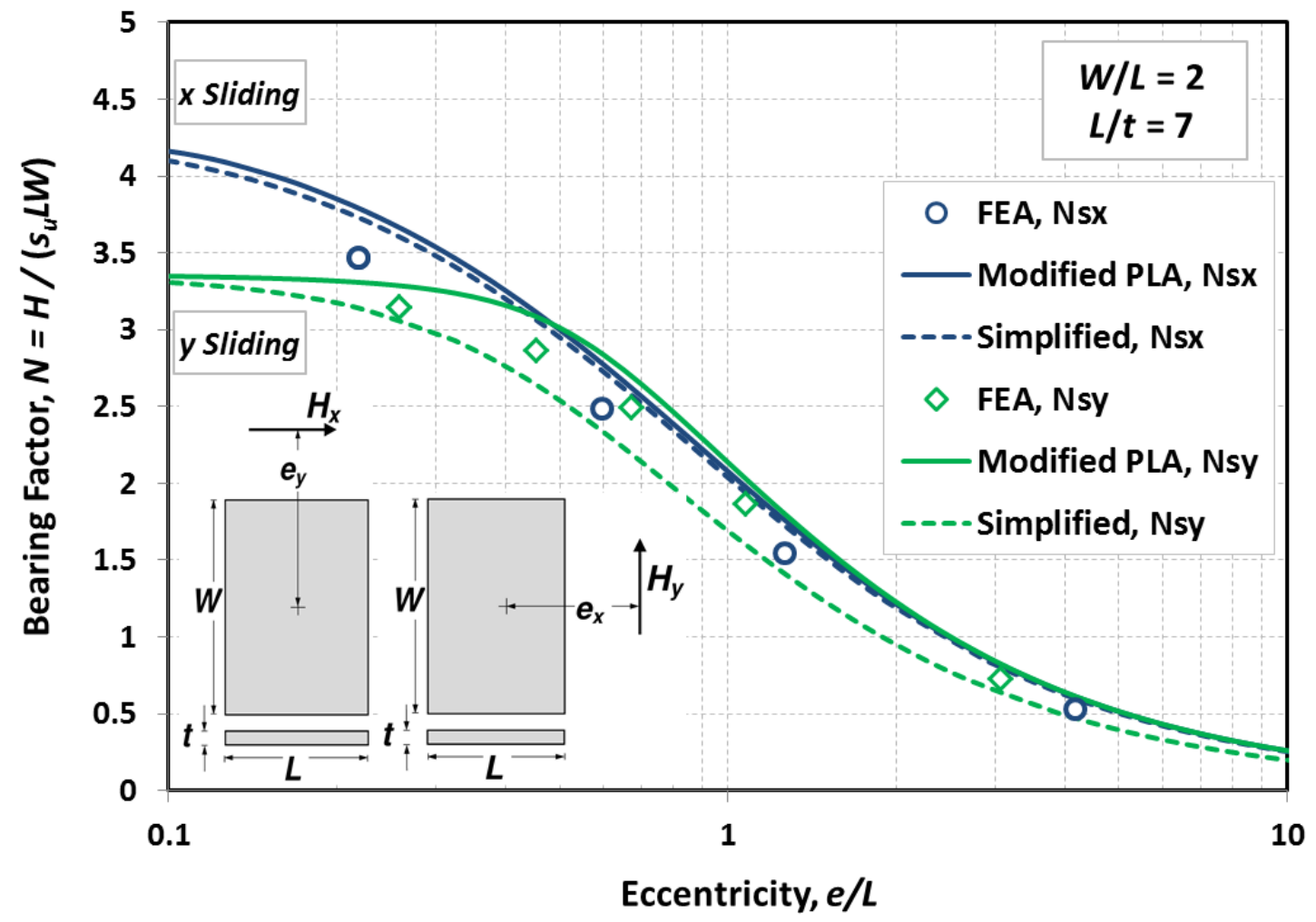

Figure 14- Reduction effect of eccentricity on sliding load capacity for rectangular $(W / L=2)$ plate of $t=L / 7$ 


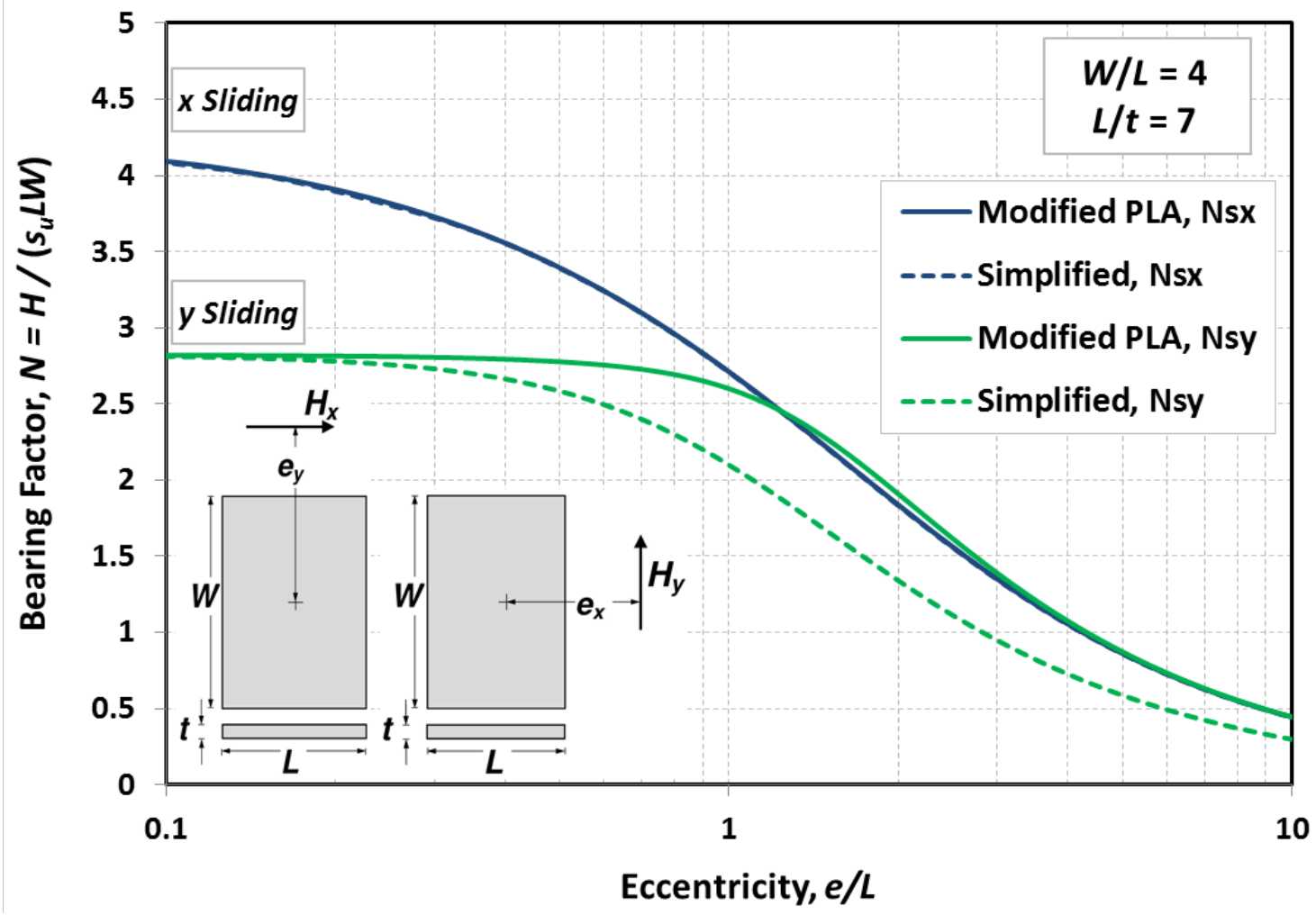

Figure 15- Reduction effect of eccentricity on sliding load capacity for rectangular $(W / L=4)$ plate of $t=L / 7$

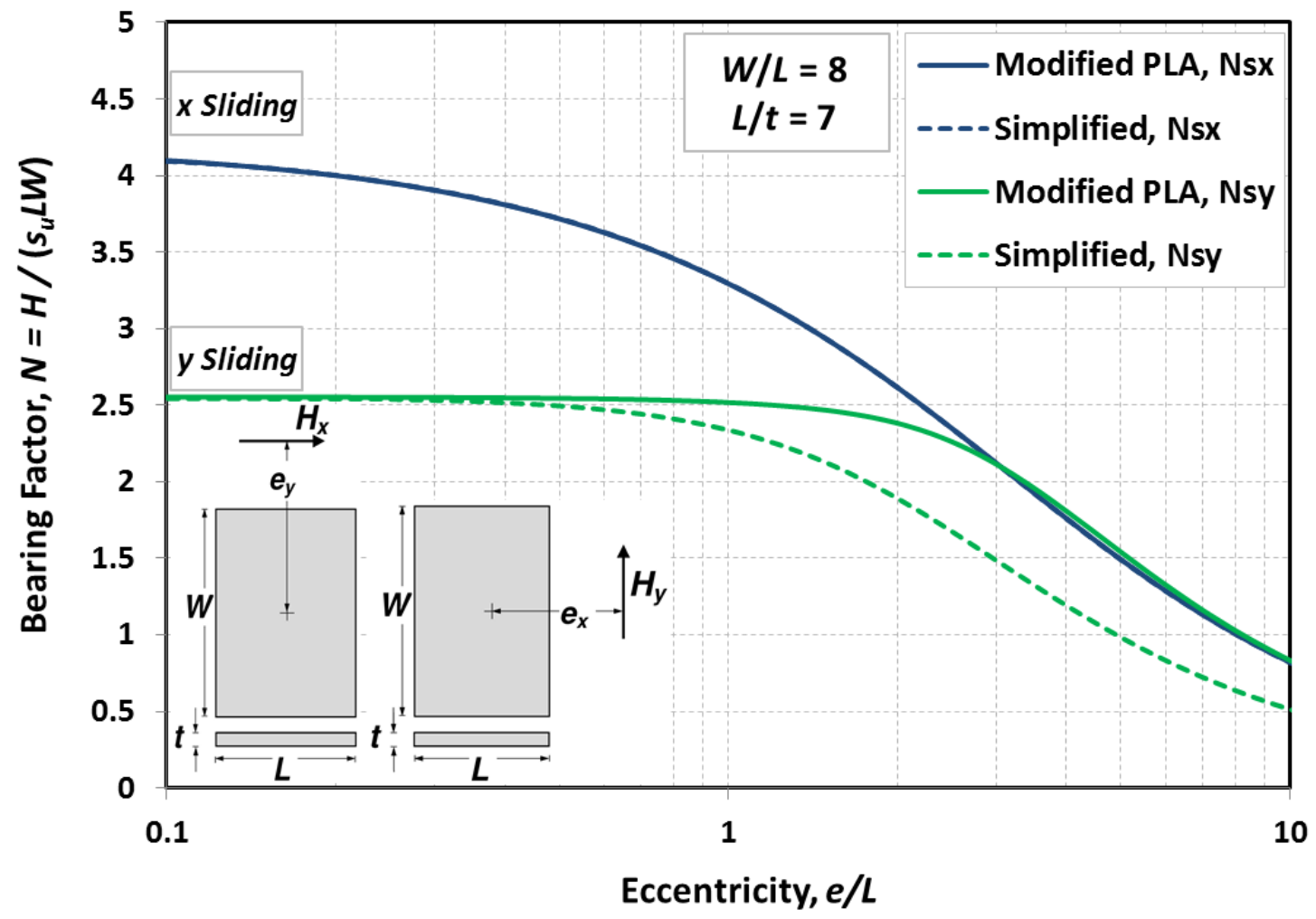

Figure 16- Reduction effect of eccentricity on sliding load capacity for rectangular $(W / L=8)$ plate of $t=L / 7$ 
As discussed in the previous sections, in most cases both simplified and modified PLA solutions

396 over-estimate the FEA predictions with increasing plate thickness except for the simplified

397 solution for rectangular plate under $y$-sliding (Fig. 14). For rectangular plates (i.e. $W / L=2,4$ and

3988 in Figs. 14-16) under eccentric $x$-sliding the PLA and simplified approaches are essentially

399 yielding the same values. As for the plate bearing capacity under $y$-sliding, the modified PLA

400 predictions show an unconservative trend compared to FEA solutions (Fig. 14), while the

401 simplified solution is on a slightly conservative side.

402 In general, the modified PLA approach provides a useful tool with minimal computational effort

403 and slightly unconservative yet fairly satisfactory predictions which is expected regarding its

404 upper bound solution and tendency to overestimate the bearing resistance for increased plate

405 thickness. However, the application of simplified method is also advisable, considering the

406 simple and convenient formulation to implement in spreadsheet applications and fairly accurate

407 results. Furthermore, there is no optimization procedure involved in the simplified solution to

408 search for a least upper bound collapse load which offers the simplified solution an advantage

409 over the modified PLA. Note that modified and PLA $A_{t=0}$ approaches generate exactly the same

410 yield envelopes for the infinitely thin plates.

411 Co-planar shear-torsion $\left(\boldsymbol{H}_{x}-\boldsymbol{H}_{y}-\mathrm{T}\right)$

412 Figs. 13-16 propose the load capacity for plates under eccentric shear load aligned with the 413 minor and major axis of the plate (i.e. $\psi=0^{\circ}$ or $90^{\circ}$ with $\gamma=0^{\circ}$ ); thus, the effect of load angle, $\gamma$, 414 and other eccentricity angles $(\psi)$ or in other words the general $H_{x}-H_{y}-T$ combination is not 415 evaluated. Impact of eccentricity angle ( $\psi$ ) was partially evaluated in Figs. 13-16. Examining the 416 effect of load angle is also clearly significant since any increase in $\gamma$ (with constant $e$ and $\psi$ ) will 
417 reduce the torsional load, so that for $\gamma=90^{\circ}$ the torsion yields to zero. Evaluating the effect of

418 load angle, $\gamma$, is possible through modified PLA solution (Eqs. 15-24).

419 The simplified solution is also applicable using the generalized PLA $A_{t=0}$ approach (Eqs. 7-10) for

420 infinitely thin plate. The simplified solution also requires the ultimate shear resistance, $H_{\max }$,

421 which is estimated based on a simple formulation summarized in Appendix C.

422

423 PLATE UPLIFT CAPACITY UNDER GENERALIZED LOADING

424 Up to this point, the bearing capacity of plate anchor subjected to combined co-planar shear load

425 and torsion has been studied. Now a question arises that how the combination of shear-torsion

426 loading affects the uplift bearing capacity of the plate anchor.

427 Throughout the paper, it was well discussed that the plate thickness does not influence the shape

428 of shear-torsion or two-way shear yield envelope in the normalized load space. We also

429 examined the shape of the normalized yield envelopes for normal-shear forces, normal force-

430 moment, and shear force-moment in the API/Deepstar 2D-FE study (Andersen et al. 2004; Murff

431 et al. 2005) for plates of $t=L / 20$ to $L / 7$. Our evaluations indicated a marginal impact of the plate

432 thickness on the shape of normalized yield surface. Therefore, application of generalized

433 interaction envelope for infinity thin plate could be extended to plate under six degrees of

434 freedom loading. The following mathematical model is adopted to describe the shape of yield

435 envelope for the plate anchor under general loading:

$$
f\left(\frac{F_{n}}{F_{n, \text { max }}}, \frac{M_{x}}{M_{x, \max }}, \frac{M_{y}}{M_{y, \text { max }}}, \frac{H_{x}}{H_{x, \text { max }}}, \frac{H_{y}}{H_{y, \text { max }}}, \frac{T}{T_{\max }}\right)=
$$

436

$$
\left(\frac{F_{n}}{F_{n, \max }}\right)^{n}+\left\{\left(\frac{M_{x}}{M_{x, \max }}\right)^{m x}+\left(\frac{M_{y}}{M_{y, \text { max }}}\right)^{m y}+\left[\left[\left(\frac{H_{x}}{H_{x, \max }}\right)^{h x}+\left(\frac{H_{y}}{H_{y, \max }}\right)^{h y}\right]^{h}+\left(\frac{T}{T_{\max }}\right)^{m z}\right]^{s}\right\}^{1 / p}-1=0
$$




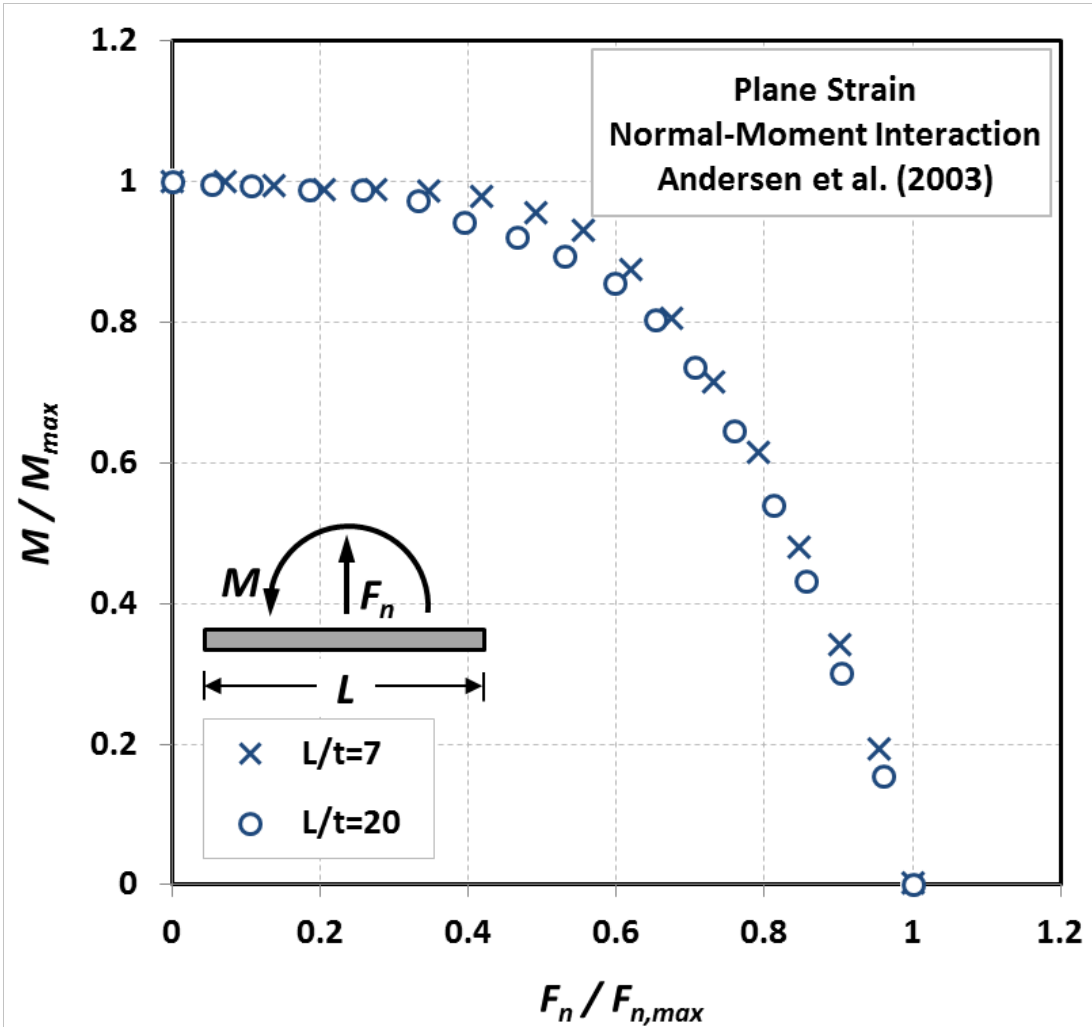

(a)

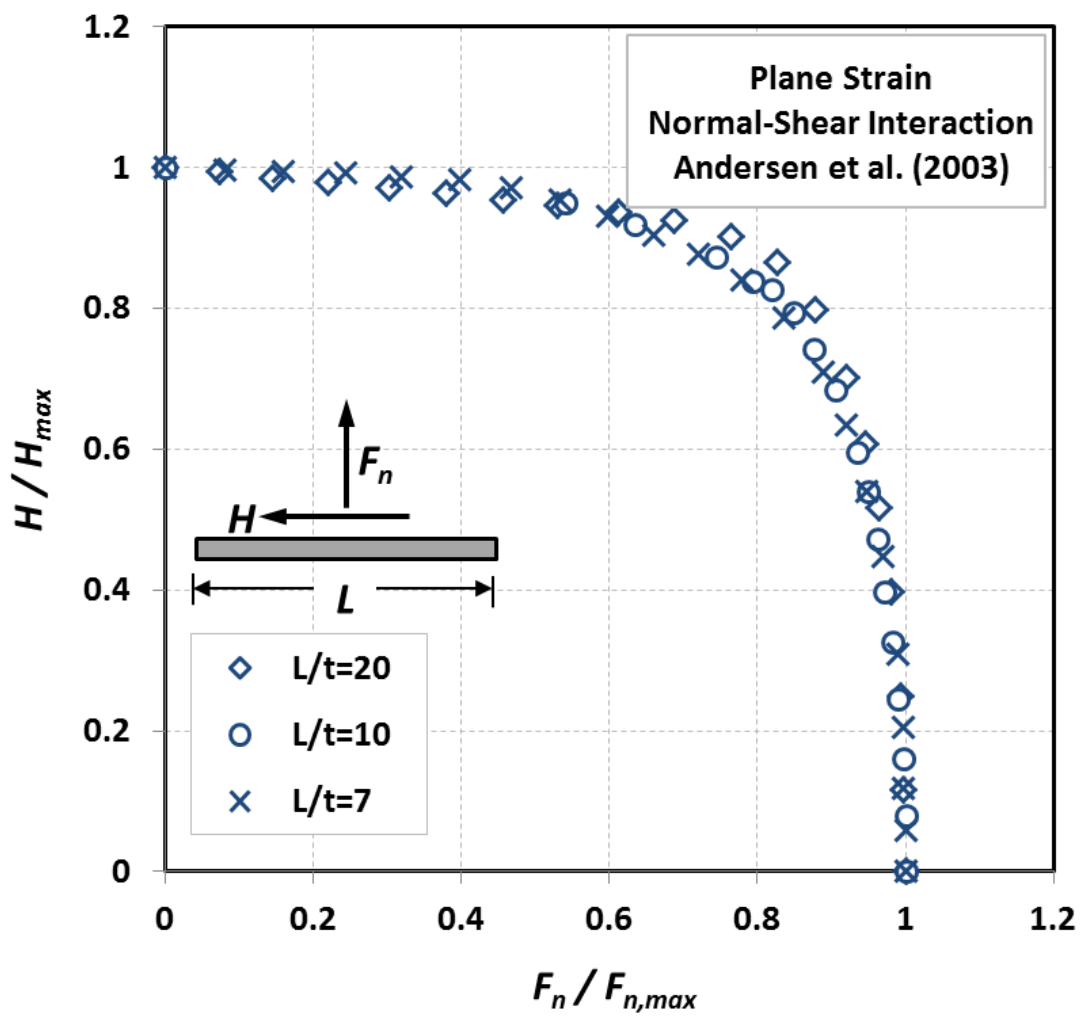

(b) 


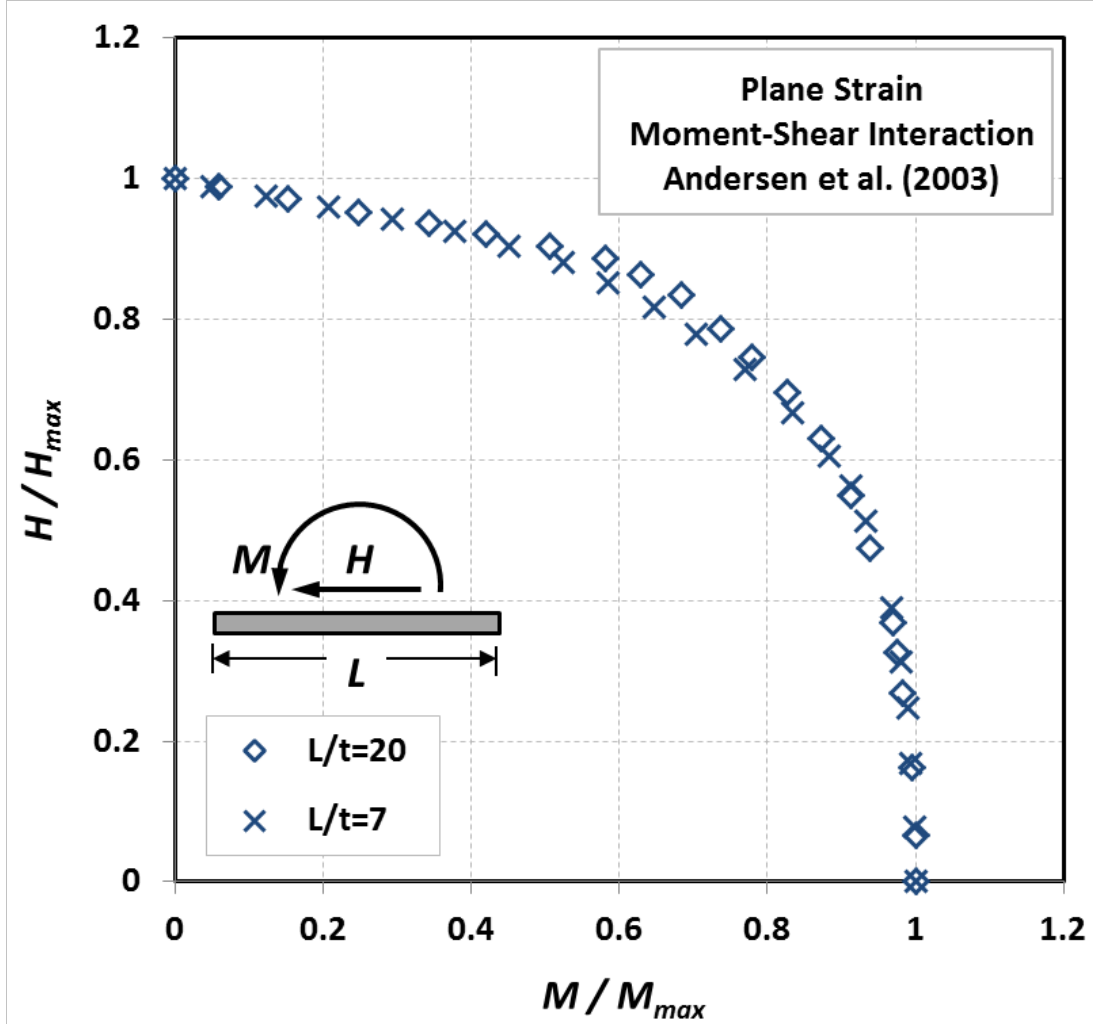

(c)

Figure 17- Yield envelopes for plane strain strip plates of thickness $t=L / 20$ to $L / 7$ (Andersen et al. 2003): (a) normal-moment; (b) normal-shear; (c) moment-shear

437 where $F_{n}, H_{x}, H_{y}=$ normal, $x$-shear, $y$ - shear forces, $M_{x}, M_{y}$, and $T: x$-moment, $y$-moment, and 438 torsion components of the generalized combined loading at failure, $F_{n, \max }, H_{x, \max }, H_{y, \max }, M_{x, \max }$, $439 M_{y, \max }$, and $T_{\max }$ are the corresponding maximum values, and eight constants of $n, m x, m y, h x, h y$, $440 \quad m z, s$, and $p$ are the interaction factors.

441 Regarding the insensitivity of the shape of normalized yield surface to plate thickness, we 442 adopted the same interaction factors proposed in Yang et al. (2010) for the infinitely thin plate. 443 Interaction factors which define the shape of yield envelope are summarized in Table 3. These 444 factors could be used for square and rectangular $(W / L=2)$ plates with any arbitrary plate thickness; 445 For other aspect ratios, Interaction factors and maximum capacity values should be evaluated. 446 Calculation of the bearing capacity for each degree-of-freedom using Eq. 26, requires all the six 
447 maximum capacity factors for the desired plate thickness and aspect ratio (rather than available

448 values) to be estimated. The interaction factors for the shear-torsion yield envelope ( $h x$, hy, and $449 \mathrm{mz}$ ) are refined regarding the present study.

Table 3. Interaction factors for generalized yield envelope

\begin{tabular}{|c|cc|}
\hline Factors & Square & Rectangular $(W / L=2)$ \\
\hline$n$ & 3.26 & 3.20 \\
\hline$m x$ & 1.91 & 1.86 \\
\hline$m y$ & 1.91 & 2.47 \\
\hline$h x$ & 2.50 & 2.50 \\
\hline$h y$ & 2.50 & 2.50 \\
\hline$h$ & 0.70 & 0.75 \\
\hline$m z$ & 1.75 & $1.15+1.23[\cos (\phi)]^{5}$ \\
\hline$s$ & 3.87 & $3.75 / m z$ \\
\hline$p$ & 1.56 & 1.93 \\
\hline
\end{tabular}

Notes:

$$
f=\left(\frac{F_{n}}{F_{n, \max }}\right)^{n}+\left\{\left(\frac{M_{x}}{M_{x, \max }}\right)^{m x}+\left(\frac{M_{y}}{M_{y, \text { max }}}\right)^{m y}+\left[\left[\left(\frac{H_{x}}{H_{x, \text { max }}}\right)^{h x}+\left(\frac{H_{y}}{H_{y, \text { max }}}\right)^{h y}\right]^{h}+\left(\frac{T}{T_{\max }}\right)^{m z}\right]^{s}\right\}^{1 / p}-1=0
$$

$\phi$ : the angle between line of action of the external force and $y$-axis $(\phi=\psi+\gamma$; Fig. 1)

451 The following relationships and recommendations also can be applied to calculate the maximum

452 capacity values for every six degrees of freedom loading. Note that the weight of plate anchor for

453 typical thickness values available in industry has a negligible effect on the capacity of the anchors:

$454 \quad$ 1- Uplift or normal ultimate capacity factor $\left(F_{n, \max }\right)$ :

455 The uplift or normal ultimate capacity of the plane strain strip plate anchor could be estimated

456 using the upper-bound derived relationship proposed by O’Neill et al. (2003):

$457 \quad N_{n, \text { max }}=\frac{F_{n, \max }}{L s_{u}}=(3 \pi+2)+2 \frac{t}{L}\left[\alpha+\frac{1+\alpha}{\sqrt{2}}\right]$ 
458 where $\alpha$ is the plate-soil adhesion factor. Table 4 also summarizes the predictions for uplift 459 capacity factor using other effective analytical and numerical approaches for 2D plane stain and $4603 \mathrm{D}$ circular, square, and rectangular plates of different thicknesses.

Table 4. Maximum pure capacity factor for deeply embedded plate anchor

\begin{tabular}{|c|c|c|}
\hline \multirow{4}{*}{ 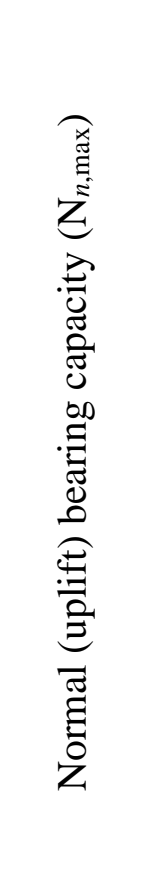 } & $\begin{array}{l}\text { 2D plane strain Lower bound PLA } \\
\text { (Rowe, 1978) }\end{array}$ & $t / L=0 ; W / L>6: 10.28$ \\
\hline & $\begin{array}{l}\text { 2D plane strain finite element } \\
\text { (Elkhatib and Randolph, 2005) }\end{array}$ & $\begin{array}{l}t / L=1 / 20 ; W / L>6: 11.62 \\
t / L=1 / 7 ; W / L>6: 11.93\end{array}$ \\
\hline & $\begin{array}{l}\text { 3D upper and lower bound PLA; } \\
\text { exact solution (Martin and } \\
\text { Randolph, 2001) }\end{array}$ & $t / L=0$; Circularr 13.11 \\
\hline & $\begin{array}{l}\text { 3D large deformation finite } \\
\text { element (LDFE) (Wang et al. } \\
\text { 2009) }\end{array}$ & $\begin{array}{c}t / L=1 / 20 ; \text { Square }(W / L=1): 13.17 \\
t / L=1 / 20 ; W / L=2: 12.35 \\
t / L=1 / 20 ; W / L=4: 11.28 \\
t / L=1 / 20 ; W / L=6: 10.95\end{array}$ \\
\hline \multirow{3}{*}{ 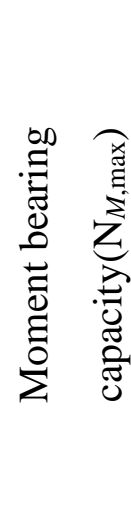 } & $\begin{array}{l}\text { 2D finite element, smooth }(\alpha=0.0) \\
\text { and rough }(\alpha=1.0) \text { strip plates } \\
\text { (Elkhatib and Randolph, 2005) }\end{array}$ & $\begin{array}{l}t / L=1 / 7: 1.44 \text { and } 1.63 \text { for } \alpha=0.0 \text { and } 1.0 \\
t / L=1 / 20: 1.41 \text { and } 1.59 \text { for } \alpha=0.0 \text { and } 1.0\end{array}$ \\
\hline & $\begin{array}{l}\text { 3D upper bound PLA (Yang et al. } \\
\text { 2010) }\end{array}$ & $t / L=0 ;$ Circular: $M_{\max }=\pi^{2} R^{3} s_{u} \Rightarrow N_{m, \max }=1.9$ \\
\hline & $\begin{array}{l}\text { Calibrated 3D finite element } \\
\text { (Yang et al. 2010) }\end{array}$ & $\begin{array}{c}t / L=0 \text {; Square }(W / L=1): N_{M x, \max }=N_{M y, \max }=1.9 \\
t / L=0 ; W / L=2: N_{M x, \max }=2.15 ; N_{M y, \max }=1.70\end{array}$ \\
\hline
\end{tabular}

Note:

1- For other plate thicknesses, the pull-out capacity factor for square shaped anchor could be used with reasonable accuracy to calculate capacity factor for circular plates with similar plate area. 
461 All the capacity factors are correct for deeply embedded plates (i.e. $H / L$ or $H / D \geq 6$ where $H$ is 462 the embedment depth, $L$ is the plate shorter length, and $D$ is the diameter of circular anchor).

463 Adopting this assumption means that the failure mechanism is localized around the plate and

464 does not extend to the surface and therefore the bearing capacity is not affected by the effective

465 overburden pressure and soil weight. Thus, the soil is assumed to be a weightless material in the

466 analysis. Also the plate and soil are fully bonded (i.e. no breakaway or separation occurs between

467 plate and soil at the time of failure). Appropriate predictions are provided by Song et al. (2008)

468 and Wang et al. (2010) to evaluate the plate pullout capacity under breakaway/separation

469 condition or when the overburden pressure is influential on the anchor capacity.

$470 \quad 2-$ Moment ultimate capacity factor $\left(M_{x, \max }, M_{y, \max }\right)$ :

471 O’Neill et al. (2003) used the plane strain upper bound plasticity solution to evaluate the

472 maximum moment capacity factor for a strip plate:

$473 \quad N_{M, \text { max }}=\frac{M_{\max }}{L^{2} s_{u}}=\frac{\pi}{2}\left[1+\left(\frac{t}{L}\right)^{2}\right]$

474 The solution could be generalized for square and rectangular plates of finite thickness to 475 calculate moment factors about $x$ and $y$ plate axis using the generalized 2D collapse mechanism 476 in 3D space (Chi 2010):

$$
N_{M x, \max }=\frac{M_{x, \max }}{W L^{2} s_{u}}=\frac{\pi}{2}\left[1+\left(\frac{t}{W}\right)^{2}\right]\left(\frac{W}{L}\right)\left\{1+\frac{1}{3}\left(\frac{W}{L}\right) \sqrt{1+\left(\frac{t}{W}\right)^{2}}\right\}
$$

$478 \quad N_{M y, \max }=\frac{M_{y, \max }}{W L^{2} s_{u}}=\frac{\pi}{2}\left[1+\left(\frac{t}{L}\right)^{2}\right]\left\{1+\frac{L}{3 W} \sqrt{1+\left(\frac{t}{L}\right)^{2}}\right\}$

479 Note that the equation for $N_{M x, \max }$ does not provide a good estimates as the $W / L$ increases, 480 probably due to the adopted failure mechanism, while the equation for $N_{M y, \max }$ works more 
481 effectively better, especially for greater $W / L$. For strip plate (i.e. $W / L \rightarrow \infty$ or $L / W \rightarrow 0$ ) Eq. (30)

482 yields the O'Neill et al (2003).

483 Some FE and PLA derived solutions for moment capacity factor are also included in Table 4 for 484 2D plane stain and 3D circular, square, and rectangular plates of different thicknesses.

485 3- Parallel or shear ultimate capacity factor $\left(N_{s x, \max }, N_{s y, \max }\right)$ :

486 As discussed previously, the limit equilibrium equations (Eqs. 2-3) provide simple yet fairly 487 accurate predictions.

488 4- Torsional ultimate capacity factor $\left(N_{t, \max }\right)$ :

489 The closed form solution developed based on limit equilibrium solution (Eqs. 5-6) with factor of $490 \quad C_{f}=0.67$ provides a fairly accurate and simple estimation for the torsional maximum capacity. 
492 The focus of this study is to offer a modified upper bound plastic limit analysis (PLA) as well as 493 a simplified baseline solution based on the limit equilibrium and virtual work approaches for 494 general conditions of shear-torsional loading. This study also evaluates the effect of eccentric 495 shear forces on the uplift capacity of a plate anchor under six-degrees-of-freedom generalized 496 loading. This study indicates the following:

497 1. Eccentricity reduces the plate shear and consequently uplift capacities. The reduction begins to become significant ( $>5 \%$ of reduction in shear capacity) in square plates at eccentricity levels of $e>0.1 L$ (Fig. 8). For perspective, an eccentricity $e / L=0.5$ - a load applied at the edge of a square plate - reduces shear capacity by more than $40 \%$.

2. Plates of higher aspect ratio $(W / L)$ are less susceptible to uplift capacity reduction due to planar eccentric loading. Reduction in uplift capacity due to eccentricity of in-plane shear loading is always more significant for shear loading parallel to the short axis of the plate.

3. The proposed baseline solutions to calculate the pure shear capacity, limit equilibrium Eqs. 2 and 3, offer fairly accurate predictions for plate under pure shear loading (Fig. 9). The limit equilibrium derived Eq. 5, to estimate pure torsional capacity for theoretical infinitely thin plate $(t=0)$, is accurate as well. For plates of finite thickness $(t>0)$, Eq. 6 offers the increase in torsional capacity induced by plate thickness which can provide reasonably accurate solutions if used in combination with a correction factor $C_{f}=0.67$. translation-torsion, significantly improves the Yang et al. (2010) PLA predictions both in terms of "size” (i.e. pure capacity values) as well as the "shape” (i.e. interaction response) of the two-way shear and shear-torsion yield envelopes. Predictions of the proposed 
modified PLA approach for the shape of shear-torsion yield envelope in normalized load space generally agree well with FEA predictions (Figs. 10-12). However, the method generally over-predicts the increase in pure torsional resistance associated with increased plate thickness, $t$ (Tables 1 and 2; Fig. 9, and Figs. 13-16). Application of the modified PLA approach is therefore advisable when the obtained normalized yield envelope is used in combination with the FEA derived or corrected pure torsional capacity (Eqs. 5 and 6) and pure shear capacity (Eqs. 2-3).

5. The general insensitivity of the shape of the shear-torsion yield loci to plate thickness $t$ (Figs. 10-12) offers the opportunity to develop a simplified analysis in which fairly accurate analytical expressions for shear resistance (Eqs. 2 and 3) are used in conjunction with a virtual work analysis for zero thickness plate (PLA $t=0$ : Eqs. 6-10) to predict the reduction in capacity due to eccentricity. The method is fairly robust, as it requires no find a least upper bound. Predictions from the simplified method provide reasonable conservative estimates of the reduction in $y$-shear resistance induced by eccentricity, however similar to modified PLA approach the reduced $x$-shear capacity factors will be over-predicted by the simplified approach, thus the results should be used with caution. generalized six-degrees-of-freedom loading to the plate thickness (Figs. 10-12, Fig. 17). Thus, to evaluate the uplift capacity reduction of a plate under any load combinations, the normalized yield surface for an infinitely thin plate (Eq. 17, Table 3) could be used in combination with the ultimate capacity factors available in the current study and literature (Table 4). 
Appendix A. Energy Dissipation Rate Terms for PLA (Fig. 2)

\begin{tabular}{|c|c|c|c|}
\hline Ref. & Mechanism & Location & Equation \\
\hline \multirow{3}{*}{ 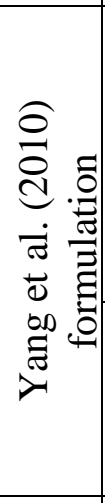 } & \multirow{2}{*}{$\begin{array}{l}\text { Slip at the } \\
\text { edges of the } \\
\text { plate }\end{array}$} & $\begin{array}{l}\text { Sides } \\
\mathrm{AD} \text { and } \\
\mathrm{BC}\end{array}$ & $\dot{D}_{e(A D, B C)}=\int_{-W / 2}^{W / 2} s_{u} \dot{\beta}\left[2 N_{e}\left|y-y_{0}\right|+\alpha\left|L / 2-x_{0}\right|+\alpha\left|L / 2+x_{0}\right|\right] t d y$ \\
\hline & & $\begin{array}{c}\text { Sides AB } \\
\text { and } C D\end{array}$ & $\dot{D}_{e(A B, C D)}=\int_{-L / 2}^{L / 2} s_{u} \dot{\beta}\left[2 N_{e}\left|x-x_{0}\right|+\alpha\left|W / 2-y_{0}\right|+\alpha\left|W / 2+y_{0}\right|\right] t d x$ \\
\hline & $\begin{array}{l}\text { Slip at the } \\
\text { top and base } \\
\text { of plate } \\
\text { ABCD }\end{array}$ & $\begin{array}{l}\text { Base and } \\
\text { top }\end{array}$ & $\dot{D}_{s}=2 \int_{-W / 2} \int_{-L / 2} \alpha s_{u} R(x, y) \dot{\beta} d x d y$ \\
\hline 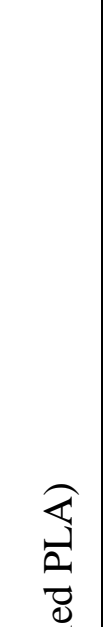 & Slip at the & $\begin{array}{l}\text { Sides } \\
\text { AD and } \\
\text { BC }\end{array}$ & $\begin{array}{l}\dot{D}_{e, B C}=\int_{-W / 2}^{W / 2} s_{u} \dot{\beta}\left(N_{s t}\left|\frac{L}{2}-x_{0}\right|+N_{n t}\left|y-y_{0}\right|\right) t d y \\
\dot{D}_{e, A D}=\int_{-W / 2}^{W / 2} s_{u} \dot{\beta}\left(N_{s t}\left|\frac{L}{2}+x_{0}\right|+N_{n t}\left|y-y_{0}\right|\right) t d y \\
\left\{\begin{array}{l}N_{s t}=N_{s t, \max }\left[\left(\frac{N_{n t, \text { max }}}{N_{s t, \text { max }}} \cdot \frac{\left|x-x_{0}\right|}{\left|y-y_{0}\right|}\right)^{2}+1\right]^{-0.5} \quad \text { where: } \begin{array}{l}x=L / 2 \text { for } B C \\
x=-L / 2 \text { for } A D\end{array} \\
N_{n t}=\left(\frac{N_{n t, \text { max }}}{N_{s t, \text { max }}}\right)^{2}\left(\frac{\left|x-x_{0}\right|}{\left|y-y_{0}\right|}\right) N_{s t}\end{array}\right.\end{array}$ \\
\hline 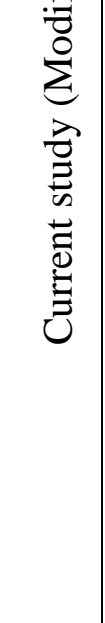 & plate & $\begin{array}{c}\text { Sides AB } \\
\text { and CD }\end{array}$ & $\begin{array}{l}\dot{D}_{e, A B}=\int_{-L / 2}^{L / 2} s_{u} \dot{\beta}\left(N_{s t}\left|\frac{W}{2}-y_{0}\right|+N_{n t}\left|x-x_{0}\right|\right) t d x \\
\dot{D}_{e, C D}=\int_{-L / 2}^{L / 2} s_{u} \dot{\beta}\left(N_{s t}\left|\frac{W}{2}+y_{0}\right|+N_{n t}\left|x-x_{0}\right|\right) t d x \\
\begin{cases}N_{s t}=N_{s t, \max }\left[\left(\frac{N_{n t, \max }}{N_{s t, \text { max }}} \cdot \frac{\left|y-y_{0}\right|}{\left|x-x_{0}\right|}\right)^{2}+1\right]^{-0.5} \quad \text { where: } \begin{array}{l}y=W / 2 \text { for } A B \\
y=-W / 2 \text { for } C D\end{array} \\
N_{n t}=\left(\frac{N_{n t, \text { max }}}{N_{s t, \text { max }}}\right)^{2}\left(\frac{\left|y-y_{0}\right|}{\left|x-x_{0}\right|}\right) N_{s t}\end{cases} \end{array}$ \\
\hline & $\begin{array}{l}\text { Slip at the } \\
\text { top and base } \\
\text { of plate } \\
\text { ABCD }\end{array}$ & $\begin{array}{l}\text { Base and } \\
\text { top }\end{array}$ & $\dot{D}_{s}=2 \int_{-W / 2}^{W / 2} \int_{-L / 2}^{L / 2} \alpha s_{u} R(x, y) \dot{\beta} d x d y$ \\
\hline
\end{tabular}


Notes:

$\left(x_{0}, y_{0}\right)$ : coordinates of the center of rotation (Fig. 2)

$\left(x_{\mathrm{f}}, y_{\mathrm{f}}\right)$ : coordinates of the application point for the external force $H$ (Fig. 2)

$s_{\mathrm{u}}$ : undrained shear strength of the soil

$L$ and $W$ : Length and width of the plate anchor parallel to $x$ and $y$ axis respectively

$\alpha$ : adhesion factor

$\dot{\beta}$ : virtual rate of rotation

$N_{e}$ : plane strain bearing capacity factor equal to 7.5

$R(x, y): \sqrt{\left(x-x_{0}\right)^{2}+\left(y-y_{0}\right)^{2}+(t / 2)^{2}}$

$N_{\text {st: }}$ normalized shear force acting along the plate edge

$N_{n t}$ : normalized force acting perpendicular to the plate edge

$N_{s t \text { max }}$ : maximum normalized shear force acting along the plate edge

$N_{n t, \max }$ : maximum normalized force acting perpendicular to the plate edge

$y$ constant for the sides $\mathrm{AB}(y=W / 2)$ and $\mathrm{CD}(y=-W / 2)$

$x$ constant for the sides BC $(x=L / 2)$ and $\mathrm{AD}(x=-L / 2)$ 


\section{APPENDIX B. Integration of dissipation rate for $\boldsymbol{d}=\mathbf{0}$ analysis}

539 Analytical evaluation of the inner integral in Eq. 12 is possible to permit a single numerical

540 integration. The resulting expression is:

$\dot{D}=2 s_{u} \dot{\beta} \int_{-W / 2}^{W / 2}\left[\left(a_{1} c_{1}-a_{2} c_{2}\right)+y^{2} \ln \left|\frac{a_{1}+c_{1}}{a_{2}+c_{2}}\right|\right] d y$

$a_{1}=\rho_{\text {opt }}+L / 2$

$541 \quad a_{2}=\rho_{\text {opt }}-L / 2$

$c_{1}=\sqrt{a_{1}^{2}+y^{2}}$

$c_{2}=\sqrt{a_{2}^{2}+y^{2}}$

542 Eq. B-1 can be integrated between the limits $-W / 2$ to $W / 2$ using classical numerical integration

543 formulas. 


\section{APPENDIX C. Estimation of $\boldsymbol{H}_{\max }$ for the simplified solution}

545 For a plate anchor subjected to horizontal load of $H$ (Fig. 1), these relationships are valid:

$$
\left(\frac{H_{x}}{H_{x, \max }}\right)^{h x}+\left(\frac{H_{y}}{H_{y, \max }}\right)^{h y}-1=0
$$

$546 \quad H_{x}=H \cdot \sin (\phi)$

$$
H_{y}=H \cdot \cos (\phi)
$$

547 where $\phi$ is the angle between line $H$ and $y$-axis (i.e. $\phi=\psi+\gamma$ in Fig. 7). $H_{x, \max }$ and $H_{y, \max }$ are

548 estimated by the limit equilibrium derived equations for pure translational load capacity using

549 Eqs. 2 and 3 which proved to be fairly accurate. As discussed previously in the paper, application

550 of the FE derived $h x=h y=2.5$ offers a fairly accurate estimate for the shape of $N_{s x}-N_{s y}$ yield

551 envelope. Therefore, $H$ for the simplified solution is evaluated using the following equation:

$552 \quad H=\left[\left(\frac{\sin (\phi)}{H_{x, \max }}\right)^{2.5}+\left(\frac{\cos (\phi)}{H_{y, \max }}\right)^{2.5}\right]^{-0.4}$ 


\section{REFERENCES}

Andersen, K.A., Murff, J.D. \& Randolph, M.F. (2004). “Deepwater Anchor Design PracticeVertically Loaded Drag Anchors”, Phase II Report to API/Deepstar JIP, Volume III.

Aubeny, CP, Murff, JD and Roesset JM (2001). “Geotechnical issues in deep and ultra deep waters,” International Journal of Geomechanics. Vol. 1, No. 2, pp. 225-247

Chen, WF, and Liu, XL (1990). Limit analysis and soil plasticity. Elsevier Publishing Co., Amsterdam, The Netherlands.

Chi, Ch (2010). Plastic limit analysis of offshore foundation and anchor. Doctoral dissertation, Texas A\&M University.

Elkhatib, S, and Randolph, MF (2005). "The effect of interface friction on the performance of drag-in plate anchors,” Proc. Int. Symp. On Frontiers in Offshore Geotechnics, IS-FOG05, Perth, pp. 171-177.

Martin, CM, and Randolph, MF (2001). “Applications of the lower and upper bound theorems of plasticity to collapse of circular foundations," Proc. 10th Int. Conf. Int. Association of Computer Methods and Advances in Geomechnics, Tucson, 2, pp. 1417-1428.

Murff, JD, Aubeny, CP, Yang, M (2010). "The effect of torsion on the sliding resistance of rectangular foundations,” Proc. $2^{\text {nd }}$ Int. Symp. on Frontiers in Offshore Geotechnics (ISFOG), Perth, Australia, pp. 439-444.

Murff, JD, Randolph, MF, Elkhatib, S, Kolk, HJ, Ruinen, RM, Strom, PJ, and Thorne, CP (2005). "Vertically loaded plate anchors for deepwater applications,” Proc. Int. Symp. on Frontiers in Offshore Geotechnics: ISFOG 2005, Perth, Australia, pp. 31-48.

Nouri, H, Biscontin, G, Aubeny, C (2014). "Undrained bearing capacity of shallow foundations under combined sliding and torsion,” ASCE Journal of Geotechnical and Geoenvironmetal 
Engineering. Vol. 140, No. 8.

Nouri, H (2013). Numerical Methods in Offshore Geotechnics: Applications to Submarine Landslides and Anchor Plates. Doctoral dissertation, Texas A\&M University.

O’Neill, MP, Bransby, MF, and Randolph, MF (2003). “Drag anchor fluke-soil interaction in clays,” Canadian Geotechnical Journal, vol. 40, pp. 78-94.

Prager, W (1959). An Introduction to Theory of Plasticity. Addison Wesley: Reading, MA.

Rowe, RK. (1978). "Soil-structure interaction analysis and its application to the prediction of anchor behavior,” PhD thesis, University of Sydney, Sydney, Australia.

Song, Z, Hu, Y, and Randolph, MF (2008). "Numerical simulation of vertical pullout of plate anchors in clay,” ASCE Journal of Geotechnical and Geoenvironmenal Engineering. Vol. 134, No. 6, pp. 866-875.

Tan, F (1990). Centrifuge and theoretical modelling of conical footings on sand. Ph.D. thesis, The University of Cambridge, Cambridge, U.K.

Wang, D, Hu, Y, and Randolph, MF (2010). “Three-dimensional large deformation finite element analysis of plate anchors in uniform clay,” ASCE Journal of Geotechnical and Geoenvironmenal Engineering. Vol. 136, No. 2, pp. 355-365.

Yang, M, Murff, JD, and Aubeny, CP (2010). “Undrained Capacity of Plate Anchors under General Loading,” ASCE Journal of Geotechnical and Geoenvironmental Engineering. Vol. 136, No. 10, pp. 1383-1393. 